\title{
Functional Group Scope in the Methylene-Free, Tandem Enyne Metathesis
}

\author{
Brian Peppers, Amol A. Kulkarni and Steven T. Diver* \\ Department of Chemistry, University at Buffalo, the State University of \\ New York, Amherst, NY, 14260-3000
}

\section{SUPPORTING INFORMATION}

\section{Table of Contents}
A. General Information S1
B. General Procedure (Terminal Alkynes) .S2
C. General Procedure (Internal Alkynes) S5
D. Cycloaddition Reactions. S8
E. ${ }^{1} \mathrm{H}$ and ${ }^{13} \mathrm{C}$ NMR Spectra for New Compounds. S10

\section{General Information}

All reactions were conducted under an argon atmosphere unless otherwise noted. Dichloromethane $\left(\mathrm{CH}_{2} \mathrm{Cl}_{2}\right)$ (alumina), benzene and toluene (alumina + Q5) were drawn from a solvent purifier (alumina) immediately prior to use. Reagent grade methanol was used for trituration and cycloaddition reaction. Ruthenium [1,3-bis-(2,4,6-trimethylphenyl)-2imidazolidinylidine]dichloro(phenylmethylene)(tricyclohexylphosphine) (Grubbs' second generation catalyst) was obtained from Materia Inc. (Pasadena, CA). 1,5Cyclooctadiene (COD) was distilled from sodium prior to use. All-cis polybutadiene was purchased from Aldrich (18137-4). Terminal alkynes $5,{ }^{1} \mathbf{7 B},{ }^{2}$ 7C, ${ }^{3} \mathbf{7 D},{ }^{4} \mathbf{7 E},{ }^{5} \mathbf{7 F},{ }^{6} \mathbf{7 G}^{7}$ and internal alkynes $\mathbf{9 C},{ }^{8} \mathbf{9 G}^{9}$ were synthesized according

\footnotetext{
${ }^{1}$ Middleton, M. D.; Diver, S. T., Tetrahedron Lett. 2005, 46, 4039-4043.

${ }^{2}$ Rodriguez, J. R.; Castedo, L.; Mascarenas, J. L., J. Org. Chem. 2000, 65, 2528-2531.

${ }^{3}$ Posner, G. H.; Weitzberg, M.; Hamill, T. G.; Asirvatham, E.; He, C. H.; Clardy, J., Tetrahedron 1986, 42, 2919-29.

${ }^{4}$ Qin, D.-G.; Zha, H.-Y.; Yao, Z.-J., J. Org. Chem. 2002, 67, 1038-1040.

${ }^{5}$ Rodriguez-Conesa, S.; Candal, P.; Jimenez, C.; Rodriguez, J., Tetrahedron Lett. 2001, 42, 6699-6702.

${ }^{6}$ Short, K. M.; Ziegler, C. B., Jr., Tetrahedron Lett. 1995, 36, 355-6.

${ }^{7}$ Luo, F. T.; Wang, R. T., Tetrahedron Lett. 1992, 33, 6835-8.
} 
to literature procedures. Alkynes $\mathbf{7 A}$ and $\mathbf{9 H}$ are commercially available from Aldrich. Column chromatography was carried out on Merck silica gel 60 (230 400 mesh). ${ }^{1} \mathrm{H}$-NMR spectra were recorded at $500 \mathrm{MHz}$ and ${ }^{13} \mathrm{C}$-NMR spectra at $125 \mathrm{MHz}$ in $\mathrm{CDCl}_{3} .{ }^{1} \mathrm{H}-\mathrm{NMR}$ spectra were referenced on the TMS signal for $\mathrm{CDCl}_{3}$. The ${ }^{13} \mathrm{C}$-NMR spectra were referenced at $77.0 \mathrm{ppm}$ for $\mathrm{CDCl}_{3}$. The syringe pump ( $k d$ scientific, model 210) was used for the tandem enyne metathesis studies using a $5 \mathrm{~mL}$ glass gas tight syringe.

\section{General Procedure (Terminal Alkynes)}

To an oven-dried $50 \mathrm{~mL}$ schlenk tube, cooled under argon, was added 1,5-cyclooctadiene ( 6 or 9 equiv) and $5 \mathrm{~mL}$ of solvent (an argon cooled coldfinger was included for all reactions performed at refluxing conditions). A rubber septum was placed on the side arm of the schlenk tube. This solution was purged with a steady stream of argon for 5 minutes via a stainless steel needle. Upon completion of argon purge, the needle was removed and a teflon cannula attached to a $5 \mathrm{~mL}$ Hamilton gas-tight syringe was passed through the septum and lowered until it reached the point where the side arm and the main body of the schlenk tube meet. The catalyst was added all at once and allowed to stir for $1 \mathrm{~min}$. The tube was then immersed in an oil bath at the specified temperature. The teflon cannula was then lowered further until resting about $2-3 \mathrm{~cm}$ above the solution line. The addition of 0.250 mmole of alkyne was then started via syringe pump at a constant rate for $4 \mathrm{~h}$. At the end of this period, the syringe was manually plunged to dispense the solution in the teflon cannula and the reaction was stirred for an additional hour. The reaction was then transferred to a $25 \mathrm{~mL}$ $\mathrm{rb}$ flask and concentrated in vacuo (rotary evaporator). A stir bar was added and the vessel was placed in an ice bath and triturated with $5 \mathrm{~mL}$ of ice-cold methanol. The trituration was performed three times. The methanol fractions where pooled and concentrated in vacuo (rotary evaporator). The resulting residue was further purified by column chromatography on silica gel, eluting with TLC solvent, as indicated for individual compounds. Depending of the efficiency of trituration and polarity of individual substrates, a second column was needed to completely remove all traces of 1,5-cyclooctadiene-derived oligomer byproducts.

Dihydrophenylalanine 6 . Solvent $=$ dichloromethane; reaction temperature $=40$ ${ }^{\circ} \mathrm{C} ; 7.5 \mathrm{~mol} \%$ complex $1 ; 2.30 \mathrm{mmol} 1,5-\mathrm{COD}$ (9 equiv). According to the general procedure $(0.256 \mathrm{mmol}$ scale) to obtain $88 \mathrm{mg} 6$ as a colorless oil ( $80 \%$ yield). ${ }^{1} \mathrm{H}$ NMR $\left(500 \mathrm{MHz}, \mathrm{CDCl}_{3}\right): \delta 7.76(\mathrm{~d}, \mathrm{~J}=7.5 \mathrm{~Hz}, 2 \mathrm{H}), 7.60,(\mathrm{~m}, 2 \mathrm{H}), 7.40(\mathrm{t}, \mathrm{J}=$ $7.0 \mathrm{~Hz}, 2 \mathrm{H}), 7.31(\mathrm{t}, \mathrm{J}=7.5 \mathrm{~Hz}, 2 \mathrm{H}), 5.83(\mathrm{~m}, 2 \mathrm{H}), 5.55(\mathrm{~s}, 1 \mathrm{H}), 5.27(\mathrm{~d}, \mathrm{~J}=8.0$

\footnotetext{
${ }^{8}$ D'Accolti, L.; Fiorentino, M.; Fusco, C.; Crupi, P.; Curci, R., Tetrahedron Lett. 2004, 45, 85758578.

${ }^{9}$ Henry, J. R.; Marcin, L. R.; McIntosh, M. C.; Scola, P. M.; Harris, G. D., Jr.; Weinreb, S. M., Tetrahedron Lett. 1989, 30, 5709-12.
} 
$\mathrm{Hz}, 1 \mathrm{H}), 4.41(\mathrm{~m}, 1 \mathrm{H}), 4.34(\mathrm{~m}, 2 \mathrm{H}), 4.23(\mathrm{~m}, 1 \mathrm{H}), 2.48(\mathrm{~m}, 2 \mathrm{H}), 2.10(\mathrm{~m}, 4 \mathrm{H})$, $1.47(\mathrm{~s}, 9 \mathrm{H}) ;{ }^{13} \mathrm{C}$ NMR $\left(125 \mathrm{MHz}, \mathrm{CDCl}_{3}\right): \delta 171.1,155.5,143.9,141.3,130.9$, 127.7, 127.3, 127.0, 126.6, 125.2, 124.4, 120.0, 82.1, 66.1, 53.6, 47.2, 38.5, 28.0, 22.3, 22.1; FT-IR (thin film, $\mathrm{cm}^{-1}$ ) 3326, 2933, 1718, 1507, 1450, 1367, 1224, 1155, 1050; high resolution ESI molecular ion calcd for $\mathrm{C}_{28} \mathrm{H}_{31} \mathrm{O}_{4} \mathrm{~N}+\mathrm{Na}$ 468.2145 found 468.2152; $[\alpha]_{D}^{25}+10.0\left(\mathrm{c}=1.6, \mathrm{CHCl}_{3}\right)$.

Table 2, entry 1 . Solvent $=$ dichloromethane; reaction temperature $=40{ }^{\circ} \mathrm{C} ; 5 \mathrm{~mol}$ $\%$ complex 1; $1.64 \mathrm{mmol} 1,5-\mathrm{COD}$ (6 equiv). According to the general procedure ( $0.274 \mathrm{mmol}$ scale) to obtain $54 \mathrm{mg} \mathbf{8 A}$ as a colorless oil ( $82 \%$ yield). $R_{f}=0.4$ (1:3 ethyl acetate-hexanes). ${ }^{1} \mathrm{H}$ NMR $\left(500 \mathrm{MHz} \mathrm{CDCl}_{3}\right) \delta 5.85$ (s, 1H), 5.77 (dd, $\mathrm{J}=1.5,10.0 \mathrm{~Hz}, 1 \mathrm{H}), 5.55(\mathrm{~s}, 1 \mathrm{H}), 3.73(\mathrm{~s}, 6 \mathrm{H}), 3.52(\mathrm{t}, \mathrm{J}=8.0 \mathrm{~Hz}, 1 \mathrm{H}), 2.63(\mathrm{~d}$, $\mathrm{J}=8.0 \mathrm{~Hz}, 2 \mathrm{H}), 2.08(\mathrm{~m}, 4 \mathrm{H}) ;{ }^{13} \mathrm{C} \operatorname{NMR}\left(125 \mathrm{MHz} \mathrm{CDCl}_{3}\right) \delta 169.4,131.9,127.6$, 126.0, 122.8, 52.4, 51.2, 34.6, 22.2, 22.0; FT-IR (thin film, $\mathrm{cm}^{-1}$ ) 3033, 2952, 2816, 1762, 1742, 1450, 1339, 1278, 1237, 1157 1031; high resolution El molecular ion calcd for $224.1043 \mathrm{C}_{12} \mathrm{H}_{16} \mathrm{O}_{4}$ found 224.1044.

Table 2, entry 2. Solvent $=$ benzene; reaction temperature $=65{ }^{\circ} \mathrm{C} ; 10 \mathrm{~mol} \%$ complex 1; $2.25 \mathrm{mmol} 1,5-\mathrm{COD}$ (9 equiv). According to the general procedure ( $0.250 \mathrm{mmol}$ scale) to obtain $73 \mathrm{mg} \mathbf{8 B}$ as a colorless oil (75\% yield). $\mathrm{R}_{f}=0.23$ (1:3 ethyl acetate-hexanes). ${ }^{1} \mathrm{H}$ NMR $\left(400 \mathrm{MHz}, \mathrm{CDCl}_{3}\right) \delta 7.93(\mathrm{~d}, \mathrm{~J}=7.6 \mathrm{~Hz}$, 4H), 7.69 (t, J = 7.2 Hz, 2H), 7.56 (t, J = $7.6 \mathrm{~Hz}, 4 \mathrm{H}), 5.77(\mathrm{~m}, 1 \mathrm{H}), 5.59$ (d, J = $10.0 \mathrm{~Hz}, 1 \mathrm{H}), 5.28(\mathrm{~s}, 1 \mathrm{H}), 4.53(\mathrm{t}, \mathrm{J}=5.2 \mathrm{~Hz}, 1 \mathrm{H}), 2.93(\mathrm{~d}, \mathrm{~J}=5.2 \mathrm{~Hz}, 2 \mathrm{H}), 1.92$ (s, 4H); ${ }^{13} \mathrm{C}$ NMR $\left(125 \mathrm{MHz}, \mathrm{CDCl}_{3}\right) \delta 138.3,134.4,129.6,129.5,129.0,128.6$, 124.4, 124.2 82.2, 31.2, 22.1, 21.6; FT-IR (thin film, $\mathrm{cm}^{-1}$ ) 3072, 3033, 2931, 2886, 2835, 1451, 1324, 1310, 1175, 1156, 1082; high resolution El molecular ion calcd for $388.0798 \mathrm{C}_{20} \mathrm{H}_{20} \mathrm{O}_{4} \mathrm{~S}_{2}$ found 388.0801

Table 2, entry 3 . Solvent $=$ dichloromethane; reaction temperature $=40{ }^{\circ} \mathrm{C} ; 7.5 \mathrm{~mol}$ $\%$ complex 1; $1.50 \mathrm{mmol} 1,5-\mathrm{COD}$ (6 equiv). According to the general procedure ( $0.250 \mathrm{mmol}$ scale) to obtain $43 \mathrm{mg} \mathbf{8 C}$ as a colorless oil (72\% yield). $R_{f}=0.15$ (1:50 ethyl acetate-hexanes). ${ }^{1} \mathrm{H}$ NMR $\left(500 \mathrm{MHz} \mathrm{CDCl}_{3}\right) \delta 5.82(\mathrm{~m}, 2 \mathrm{H}), 5.52$ (s, $1 \mathrm{H}), 3.64$ (t, J = 7.0 Hz, 2H), 2.24 (t, J = 7.0 Hz, 2H), 2.09 (m, 4H), $0.89(\mathrm{~s}, 9 \mathrm{H})$, $0.043(\mathrm{~s}, 6 \mathrm{H}) ;{ }^{13} \mathrm{C}$ NMR $\left(125 \mathrm{MHz}, \mathrm{CDCl}_{3}\right) \delta 132.9,127.4,126.5,122.1,62.9$, 39.2, 26.0, 22.4, 22.2, 18.4, -5.3; FT-IR (thin film, $\mathrm{cm}^{-1}$ ) 2927, 2851, 1470, 1258, 1106, 845; high resolution El molecular ion calcd for $238.1747 \mathrm{C}_{14} \mathrm{H}_{26} \mathrm{O}_{1} \mathrm{Si}_{1}$ found 238.1748

Table 2, entry 4. Solvent $=$ dichloromethane; reaction temperature $=40{ }^{\circ} \mathrm{C} ; 7.5 \mathrm{~mol}$ $\%$ complex 1; $2.25 \mathrm{mmol} 1,5-\mathrm{COD}$ (9 equiv). According to the general procedure (0.250 mmol scale) to obtain $37.5 \mathrm{mg}$ 8D as a colorless oil (70\% yield). $R_{f}=0.19$ (1:25 ethyl acetate-hexanes). ${ }^{1} \mathrm{H}$ NMR (500 MHz, $\left.\mathrm{CDCl}_{3}\right) \delta 7.33(\mathrm{~m}, 4 \mathrm{H}), 7.27$ (m, 1H), $5.83(\mathrm{~m}, 2 \mathrm{H}), 5.56(\mathrm{~S}, 1 \mathrm{H}), 4.52(\mathrm{~s}, 2 \mathrm{H}), 3.53$, (t, J = 7.0 Hz, 2H), 2.35 (t, $\mathrm{J}=7.0 \mathrm{~Hz}, 2 \mathrm{H}), 2.10(\mathrm{~m}, 4 \mathrm{H}) ;{ }^{13} \mathrm{C}$ NMR $\left(125 \mathrm{MHz} \mathrm{CDCl}_{3}\right) \delta 138.6,132.7,128.3$, 
127.6, 127.5, 127.1, 126.7, 121.9, 72.9, 69.5, 35.9, 35.9, 22.4, 22.2; FT-IR (thin film, $\mathrm{cm}^{-1}$ ) 3036, 2931, 2862, 1109, 737; high resolution El molecular ion calcd for $214.1352 \mathrm{C}_{15} \mathrm{H}_{18} \mathrm{O}$ found 214.1355

Table 2, entry 5. Solvent $=$ dichloromethane; reaction temperature $=40^{\circ} \mathrm{C} ; 5.0 \mathrm{~mol}$ $\%$ complex $1 ; 1.50 \mathrm{mmol} 1,5-\mathrm{COD}$ (6 equiv). According to the general procedure $\left(0.250 \mathrm{mmol}\right.$ scale) to obtain $50.0 \mathrm{mg} 8 \mathrm{E}$ as a colorless oil $\left(72 \%\right.$ yield). $\mathrm{R}_{f}=0.19$ (1:25 ethyl acetate-hexanes). ${ }^{1} \mathrm{H}$ NMR $\left(500 \mathrm{MHz}, \mathrm{CDCl}_{3}\right) \delta 7.78(\mathrm{~d}, \mathrm{~J}=8.0 \mathrm{~Hz}$, 2H), $7.34(\mathrm{~d}, \mathrm{~J}=8.0 \mathrm{~Hz}, 2 \mathrm{H}), 5.80(\mathrm{~m}, 1 \mathrm{H}), 5.68(\mathrm{~d}, \mathrm{~J}=8.8 \mathrm{~Hz}, 1 \mathrm{H}), 5.49(\mathrm{~s}, 1 \mathrm{H})$, 4.04 (t, J = 6.8 Hz, 2H), $2.45(\mathrm{~s}, 3 \mathrm{H}), 2.36(\mathrm{t}, \mathrm{J}=6.8 \mathrm{~Hz}, 2 \mathrm{H}), 2.06(\mathrm{~m}, 4 \mathrm{H}) ;{ }^{13} \mathrm{C}$ NMR $\left(125 \mathrm{MHz}, \mathrm{CDCl}_{3}\right) \delta 144.6,133.2,130.4,129.7,127.8,127.4,126.1,123.3$, 69.3, 34.9, 22.2, 21.9, 21.6; FT-IR (thin film, $\mathrm{cm}^{-1}$ ) 2920, 1603, 1365, 1175, 966; high resolution ESI molecular ion calcd for $301.0869 \mathrm{C}_{15} \mathrm{H}_{18} \mathrm{O}_{3} \mathrm{NaS}$ found 301.0862

Table 2, entry 6. Solvent $=$ dichloromethane; reaction temperature $=40{ }^{\circ} \mathrm{C} ; 5.00$ mol \% complex 1; $2.25 \mathrm{mmol} 1,5-\mathrm{COD}$ (9 equiv). According to the general procedure $(0.250 \mathrm{mmol}$ scale) to obtain $43.3 \mathrm{mg} \mathbf{8 F}$ as a colorless oil $(73 \%$ yield). $\mathrm{R}_{f}=0.15$ (1:5 ethyl acetate-hexanes). ${ }^{1} \mathrm{H}$ NMR $\left(500 \mathrm{MHz}, \mathrm{CDCl}_{3}\right) \delta 7.73(\mathrm{~d}, \mathrm{~J}=$ $10 \mathrm{~Hz}, 2 \mathrm{H}$ ), $7.30(\mathrm{~d}, \mathrm{~J}=10.0 \mathrm{~Hz}, 2 \mathrm{H}), 5.80(\mathrm{~m}, 1 \mathrm{H}), 5.63(\mathrm{dd}, \mathrm{J}=1.5,10.0 \mathrm{~Hz}$, $1 \mathrm{H}), 5.45(\mathrm{~s}, 1 \mathrm{H}), 4.46(\mathrm{t}, \mathrm{J}=6.0 \mathrm{~Hz}, 1 \mathrm{H}), 3.00(\mathrm{q}, \mathrm{J}=6.5 \mathrm{~Hz}, 2 \mathrm{H}), 2.43(\mathrm{~s}, 3 \mathrm{H})$, $2.14(\mathrm{t}, \mathrm{J}=6.5,2 \mathrm{H}), 2.07(\mathrm{~m}, 4 \mathrm{H}) ;{ }^{13} \mathrm{C}$ NMR $\left(125 \mathrm{MHz}, \mathrm{CDCl}_{3}\right) \delta 143.3,137.0$, 131.6, 129.6, 128.0, 127.1, 125.6, 123.4, 41.3, 35.1, 22.2, 22.1, 21.5; FT-IR (thin film, $\left.\mathrm{cm}^{-1}\right)$ 3282, 2940, 2874, 2822, 1427, 1326, 1159, 1092; high resolution ESI molecular ion calcd for $300.1029 \mathrm{C}_{15} \mathrm{H}_{19} \mathrm{O}_{2} \mathrm{NNaS}$ found 300.1032

Table 2, entry 7. Solvent $=$ dichloromethane; reaction temperature $=40^{\circ} \mathrm{C} ; 5.0 \mathrm{~mol}$ $\%$ complex $1 ; 2.25 \mathrm{mmol} 1,5-\mathrm{COD}$ (9 equiv). According to the general procedure $\left(0.250 \mathrm{mmol}\right.$ scale) to obtain $54.0 \mathrm{mg} \mathbf{8 G}$ as a colorless oil $(74 \%$ yield $) . \mathrm{R}_{f}=0.19$ (1:5 ethyl acetate-hexanes). ${ }^{1} \mathrm{H}$ NMR $\left(500 \mathrm{MHz}, \mathrm{CDCl}_{3}\right) \delta 7.75(\mathrm{~d}, \mathrm{~J}=8.5 \mathrm{~Hz}$, 2H), $7.30(\mathrm{~d}, \mathrm{~J}=8.0 \mathrm{~Hz}, 2 \mathrm{H}), 5.81(\mathrm{~m}, 1 \mathrm{H}), 5.69(\mathrm{~d}, \mathrm{~J}=8.5 \mathrm{~Hz}, 1 \mathrm{H}), 5.39(\mathrm{~s}, 1 \mathrm{H})$, 4.60 (t, J = 6.5 Hz, 1H), $2.92(\mathrm{q}, \mathrm{J}=6.5 \mathrm{~Hz}, 2 \mathrm{H}), 2.43(\mathrm{~s}, 3 \mathrm{H}), 2.05(\mathrm{~s}, 4 \mathrm{H}), 1.99$ (t, J = 7.0 Hz, 2H), $1.56(\mathrm{dt}, \mathrm{J}=7.0,14.5 \mathrm{~Hz}, 2 \mathrm{H}) ;{ }^{13} \mathrm{C} \mathrm{NMR}\left(125 \mathrm{MHz}, \mathrm{CDCl}_{3}\right) \delta$ 143.3, 137.0, 134.3, 129.6, 127.3, 127.1, 126.5, 121.0, 42.6, 32.4, 27.9, 22.3, 22.2, 21.5; FT-IR (thin film, $\mathrm{cm}^{-1}$ ) 3280, 3039, 2934, 2834, 2828, 1429, 1325, 1159 , 1092; high resolution ESI molecular ion calcd for $292.1366 \mathrm{C}_{16} \mathrm{H}_{22} \mathrm{O}_{2} \mathrm{NS}$ found 292.1369 .

Alkyne 7H (substrate for table 1, entry 8). To a solution of 2-hydroxypent4-ynoic acid $^{10}(500 \mathrm{mg}, 3.9 \mathrm{mmol})$ and tert-butyldimethylsilylchloride $(647 \mathrm{mg}$,

\footnotetext{
${ }^{10}$ Dragovich, P. S.; Prins, T. J.; Zhou, R.; Johnson, T. O.; Hua, Y.; Luu, H. T.; Sakata, S. K.; Brown, E. L.; Maldonado, F. C.; Tuntland, T.; Lee, C. A.; Fuhrman, S. A.; Zalman, L. S.; Patick, A. K.; Matthews, D. A.; Wu, E. Y.; Guo, M.; Borer, B. C.; Nayyar, N. K.; Moran, T.; Chen, L.; Rejto, P. A.; Rose, P. W.; Guzman, M. C.; Dovalsantos, E. Z.; Lee, S.; McGee, K.; Mohajeri, M.; Liese,
} 
$4.29 \mathrm{mmol})$ in dichloromethane $(16 \mathrm{~mL})$ was added imidazole $(664 \mathrm{mg}, 9.75$ $\mathrm{mmol})$. After 12 hours the reaction was washed with $20 \mathrm{~mL}$ of saturated ammonium chloride; extracted with dichloromethane $(2 \times 20 \mathrm{~mL})$. The organic layers were combined and washed with $20 \mathrm{~mL}$ of brine and dried over anhydrous sodium sulfate. After filtration and concentration in vacuo (rotary evaporator) the residue was further purified by column chromatography on silica gel, eluting with 1:20 ethyl acetate-hexanes to give $745 \mathrm{mg}$ of $\mathbf{7 H}$ ( $79 \%$ yield). $\mathbf{R}_{f}=0.18$ (1:25 ethyl acetate-hexanes). ${ }^{1} \mathrm{H}$ NMR $\left(400 \mathrm{MHz}, \mathrm{CDCl}_{3}\right) \delta 4.03$ (dd, $\mathrm{J}=5.0,2.4 \mathrm{~Hz}$, $1 \mathrm{H}), 3.71(\mathrm{~S}, 3 \mathrm{H}), 2.62(\mathrm{ddd}, \mathrm{J}=\mathrm{Hz}, 1 \mathrm{H}), 2.62(\mathrm{~m}, 1 \mathrm{H}), 2.52(\mathrm{~m}, 1 \mathrm{H}), 1.98(\mathrm{t}, \mathrm{J}=$ $2.8 \mathrm{~Hz}, 1 \mathrm{H}), 0.87(\mathrm{~s}, 9 \mathrm{H}), 0.07(\mathrm{~d}, \mathrm{~J}=2.0 \mathrm{~Hz}, 6 \mathrm{H}) ;{ }^{13} \mathrm{C} \mathrm{NMR}\left(125 \mathrm{MHz}, \mathrm{CDCl}_{3}\right) \delta$ 172.3, 79.9, 71.1, 70.5, 52.0, 25.6, 25.5, 18.3, -5.1, -5.3; FT-IR (thin film, $\mathrm{cm}^{-1}$ ) 3321, 3296, 2963, 2931, 2854, 1760, 1472, 1267, 1146, 941, 839, 788; high resolution ESI molecular ion calcd for $265.1230 \mathrm{C}_{12} \mathrm{H}_{22} \mathrm{O}_{3} \mathrm{NaSi}$ found 265.1227.

Table 2, entry 8. Solvent $=$ dichloromethane; reaction temperature $=40{ }^{\circ} \mathrm{C} ; 10.0$ mol \% complex 1; $2.25 \mathrm{mmol} 1,5-\mathrm{COD}$ (9 equiv). According to the general procedure $(0.250 \mathrm{mmol}$ scale) to obtain $44.3 \mathrm{mg} \mathbf{8 H}$ as a colorless oil $(60 \%$ yield). $\mathrm{R}_{f}=0.25$ (1:25 ethyl acetate-hexanes). ${ }^{1} \mathrm{H}$ NMR $\left(400 \mathrm{MHz}, \mathrm{CDCl}_{3}\right) \delta 5.81$ (m, $2 \mathrm{H}), 5.54(\mathrm{~s}, 1 \mathrm{H}), 4.19(\mathrm{dd}, \mathrm{J}=4.0,8.8 \mathrm{~Hz} 1 \mathrm{H}), 3.69(\mathrm{~s}, 3 \mathrm{H}) 2.41$ (dd, J = 3.2, 13.2 Hz, 1H), $2.30(\mathrm{dd}, \mathrm{J}=8.8,13.6 \mathrm{~Hz}, 1 \mathrm{H}), 2.07(\mathrm{~m}, 4 \mathrm{H}), 0.85(\mathrm{~s}, 9 \mathrm{H}), 0.00(\mathrm{~d}$, $6 \mathrm{H}) ;{ }^{13} \mathrm{C}$ NMR $\left(125 \mathrm{MHz}, \mathrm{CDCl}_{3}\right) \delta 173.9,131.4,127.1,126.7,124.1,51.7,41.7$, 25.7, 22.4, 22.1, 18.3, -5.2, -5.3; FT-IR (thin film, $\mathrm{cm}^{-1}$ ) 2931, 2863, 1762, 1738, $1439,1259,1141,842,780$; high resolution ESI molecular ion calcd for 319.1700 $\mathrm{C}_{16} \mathrm{H}_{28} \mathrm{O}_{3} \mathrm{NaSi}$ found 319.1698

\section{General Procedure (Internal Alkynes)}

To an oven-dried $50 \mathrm{~mL}$ schlenk tube, cooled under argon, was added 1,5-cyclooctadiene (9 equiv) and $5 \mathrm{~mL}$ of solvent (an argon cooled cold-finger was included for all the reactions performed). A rubber septum was placed on the side arm of the schlenk tube. This solution was purged with a steady stream of argon for 5 minutes via a stainless steel needle. Upon completion of argon purge, the needle was removed and a teflon cannula attached to a $5 \mathrm{~mL}$ Hamilton gas-tight syringe was passed through the septum and lowered until it reached the point where the side arm and the main body of the schlenk tube meet. The catalyst was added all at once and allowed to stir for $1 \mathrm{~min}$. The tube was then immersed in an oil bath at the specified temperature. The Teflon cannula was then lowered further until resting about $2-3 \mathrm{~cm}$ above the solution line. The addition of 0.250 mmole of alkyne was then started via syringe pump at a constant rate for $4 \mathrm{~h}$. At the end of this period, the syringe was manually plunged to dispense the solution in the teflon cannula and the reaction was stirred for an additional hour. The reaction was then transferred to a $25 \mathrm{~mL} \mathrm{rb}$ flask and concentrated in vacuo (rotary evaporator). A stir bar was added and

A.; Tao, J.; Kosa, M. B.; Liu, B.; Batugo, M. R.; Gleeson, J.-P. R.; Wu, Z. P.; Liu, J.; Meador, J.

W., III; Ferre, R. A., J. Med. Chem. 2003, 46, 4572-4585. 
the vessel was placed in an ice bath and triturated with $10 \mathrm{~mL}$ of ice-cold methanol. The trituration was performed three times. The methanol fractions where pooled and concentrated in vacuo (rotary evaporator). The resulting residue was further purified by column chromatography on silica gel, eluting with TLC solvent, as indicated for individual compounds. Depending of the efficiency of trituration and polarity of individual substrates, a second column was needed to completely remove all traces of 1,5-cyclooctadiene-derived oligomer byproducts.

Table 3, entry 1 . Solvent $=$ dichloromethane; reaction temperature $=40{ }^{\circ} \mathrm{C} ; 5 \mathrm{~mol}$ $\%$ complex 1; $2.25 \mathrm{mmol} 1,5-\mathrm{COD}$ (9 equiv). According to the general procedure to obtain $40 \mathrm{mg} 10 \mathrm{~A}$ as a pale yellow oil $\left(65 \%\right.$ yield). $R_{f} 0.31$ (10\% ethyl acetate in hexanes). ${ }^{1} \mathrm{H}-\mathrm{NMR}\left(500 \mathrm{MHz}, \mathrm{CDCl}_{3}, \mathrm{ppm}\right) \delta 8.05(\mathrm{~d}, \mathrm{~J}=8.0 \mathrm{~Hz}, 2 \mathrm{H}), 7.55(\mathrm{t}, \mathrm{J}$ $=8.0 \mathrm{~Hz}, 1 \mathrm{H}), 7.44(\mathrm{t}, \mathrm{J}=8.0 \mathrm{~Hz}, 2 \mathrm{H}), 6.02(\mathrm{~s}, 1 \mathrm{H}), 5.64(\mathrm{~s}, 1 \mathrm{H}), 4.88(\mathrm{~s}, 2 \mathrm{H})$, $2.20(\mathrm{q}, \mathrm{J}=7.5 \mathrm{~Hz}, 2 \mathrm{H}), 2.16-2.10(\mathrm{~m}, 4 \mathrm{H}), 1.07$ (t, J = $7.5 \mathrm{~Hz}, 3 \mathrm{H}) ;{ }^{13} \mathrm{C}-\mathrm{NMR}$ $\left(125 \mathrm{MHz}, \mathrm{CDCl}_{3}, \mathrm{ppm}\right) \delta 166.4,137.1,132.9,132.7,130.4,129.6,128.3,128.1$, 121.2, 65.9, 24.7, 22.6, 22.2, 13.0; FT-IR (thin film, $\mathrm{cm}^{-1}$ ) 2934, 1718, 1269, 1109, 710; High-resolution $\mathrm{MS}\left(\mathrm{El}^{+}, \mathrm{m} / \mathrm{z}\right)$ molecular ion calcd for $\mathrm{C}_{16} \mathrm{H}_{18} \mathrm{O}_{2}$ 242.1301, found 242.1310, error $3.7 \mathrm{ppm}$.

Table 3, entry 2. 10B was synthesized according to the general procedure $(0.250$ mmol scale) to obtain $36 \mathrm{mg} \mathrm{10B}$ as a pale yellow oil (59\% yield). $R_{f}=0.32(10$ $\%$ ethyl acetate in hexanes). ${ }^{1} \mathrm{H}-\mathrm{NMR}\left(500 \mathrm{MHz}, \mathrm{CDCl}_{3}, \mathrm{ppm}\right) \delta 8.04$ (d, J = 7.5 $\mathrm{Hz}, 2 \mathrm{H}$ ), 7.55 (t, J = $7.5 \mathrm{~Hz}, 1 \mathrm{H}$ ), 7.43 (t, J = $7.5 \mathrm{~Hz}, 2 \mathrm{H}$ ), 5.69 (s, 1H), 5.62 (s, $1 \mathrm{H}), 4.36(\mathrm{t}, \mathrm{J}=7.5 \mathrm{~Hz}, 2 \mathrm{H}), 2.57(\mathrm{t}, \mathrm{J}=7.5 \mathrm{~Hz}, 2 \mathrm{H}), 2.06-2.03(\mathrm{~m}, 4 \mathrm{H}), 1.85(\mathrm{~s}$, $3 \mathrm{H}) ;{ }^{13} \mathrm{C}-\mathrm{NMR}\left(125 \mathrm{MHz}, \mathrm{CDCl}_{3}, \mathrm{ppm}\right) \delta 166.5,133.6,132.8,132.5,130.4,129.5$, 128.3, 124.0, 123.2, 64.4, 32.1, 22.8, 22.6, 19.6; FT-IR (thin film, $\mathrm{cm}^{-1}$ ) 2935, $1719,1272,1112,1027,710$; High-resolution $\mathrm{MS}\left(\mathrm{El}^{+}, \mathrm{m} / \mathrm{z}\right)$ molecular ion calcd for $\mathrm{C}_{16} \mathrm{H}_{18} \mathrm{O}_{2} 242.1301$, found 242.1308, error $3.0 \mathrm{ppm}$.

Table 3, entry 3. Solvent $=$ dichloromethane; reaction temperature $=40{ }^{\circ} \mathrm{C} ; 5 \mathrm{~mol}$ $\%$ complex 1; 9x0.50 mmol 1,5-COD (9 equiv). According to the general procedure $(0.50 \mathrm{mmol}$ scale) to obtain $71 \mathrm{mg} 10 \mathrm{C}$ as a pale yellow oil $(63 \%$ yield). $\mathrm{R}_{f}=0.34$ (25\% ethyl acetate in hexanes). ${ }^{1} \mathrm{H}-\mathrm{NMR}\left(500 \mathrm{MHz}, \mathrm{CDCl}_{3}\right.$, ppm) $\delta 5.89(\mathrm{~s}, 2 \mathrm{H}), 4.55(\mathrm{~s}, 4 \mathrm{H}), 2.11(\mathrm{~d}, \mathrm{~J}=1.5 \mathrm{~Hz}, 4 \mathrm{H}), 1.98(\mathrm{~s}, 6 \mathrm{H}) ;{ }^{13} \mathrm{C}-\mathrm{NMR}$ $\left(125 \mathrm{MHz}, \mathrm{CDCl}_{3}, \mathrm{ppm}\right) \delta 170.4,130.4,128.9,65.0,21.8,20.8$; FT-IR (thin film, $\left.\mathrm{cm}^{-1}\right)$ 2939, 1740, 1378, 1230, 1025, 964; High-resolution $\mathrm{MS}\left(\mathrm{El}^{+}, \mathrm{m} / \mathrm{z}\right)$ molecular ion calcd for $\mathrm{C}_{12} \mathrm{H}_{16} \mathrm{O}_{4} 224.1043$, found 224.1050, error $3.3 \mathrm{ppm}$.

Table 3, entry 4. Solvent $=$ dichloromethane; reaction temperature $=40{ }^{\circ} \mathrm{C} ; 10 \mathrm{~mol}$ $\%$ complex 1; 9x1.00 mmol 1,5-COD (9 equiv). According to the general procedure (1.00 mmol scale) to obtain $175 \mathrm{mg}$ 10D as a pale yellow oil $(68 \%$ yield). $\mathrm{R}_{f}=0.36$ (10\% ethyl acetate in hexanes). ${ }^{1} \mathrm{H}-\mathrm{NMR}\left(500 \mathrm{MHz}, \mathrm{CDCl}_{3}\right.$, ppm) $\delta 7.26(\mathrm{t}, \mathrm{J}=7.5 \mathrm{~Hz}, 2 \mathrm{H}), 7.22-7.16(\mathrm{~m}, 3 \mathrm{H}), 5.78(\mathrm{~s}, 1 \mathrm{H}), 5.61(\mathrm{~s}, 1 \mathrm{H})$, 
5.56 (t, J = 6.0 Hz, 1H), 2.94 (dd, J = 14.0, $6.0 \mathrm{~Hz}, 1 \mathrm{H}$ ), 2.88 (dd, J = 14.0, 6.0 $\mathrm{Hz}, 1 \mathrm{H}), 2.05-2.01(\mathrm{~m}, 4 \mathrm{H}), 2.00(\mathrm{~s}, 3 \mathrm{H}), 1.85$ (s, 3H); ${ }^{13} \mathrm{C}-\mathrm{NMR}\left(125 \mathrm{MHz}, \mathrm{CDCl}_{3}\right.$, ppm) $\delta 170.0,137.5,136.9,131.1,129.5,128.1,126.4,124.0,123.3,74.2,41.4$, 22.3, 22.2, 21.2, 19.7; FT-IR (thin film, $\mathrm{cm}^{-1}$ ) 2929, 1742, 1369, 1235, 1020; Highresolution $\mathrm{MS}\left(\mathrm{El}^{+}, \mathrm{m} / \mathrm{z}\right)$ molecular ion calcd for $\mathrm{C}_{17} \mathrm{H}_{20} \mathrm{O}_{2} 256.1458$, found 256.1459, error $0.4 \mathrm{ppm}$.

Table 3, entry 5 . Solvent $=$ benzene; reaction temperature $=65{ }^{\circ} \mathrm{C} ; 10 \mathrm{~mol} \%$ complex 1; $9 \times 0.25 \mathrm{mmol} 1,5-\mathrm{COD}$ (9 equiv). According to the general procedure $\left(0.250 \mathrm{mmol}\right.$ scale) to obtain $34 \mathrm{mg} 10 \mathrm{E}$ as a colorless oil (53\% yield). $\mathrm{R}_{f}=0.54$ (10\% ethyl acetate in hexanes). ${ }^{1} \mathrm{H}-\mathrm{NMR}\left(500 \mathrm{MHz}, \mathrm{CDCl}_{3}, \mathrm{ppm}\right) \delta 5.59-5.56$ $(\mathrm{m}, 2 \mathrm{H}), 3.63(\mathrm{t}, \mathrm{J}=7.5 \mathrm{~Hz}, 2 \mathrm{H}), 2.33(\mathrm{t}, \mathrm{J}=7.5 \mathrm{~Hz}, 2 \mathrm{H}), 2.05-1.98(\mathrm{~m}, 4 \mathrm{H})$, 1.79 (s, 3H), 0.90 (s, 9H), 0.05 (s, 6H); ${ }^{13} \mathrm{C}-\mathrm{NMR}\left(125 \mathrm{MHz}, \mathrm{CDCl}_{3}, \mathrm{ppm}\right) \delta$ 134.6, 131.1, 123.5, 122.6, 63.6, 36.5, 26.0, 22.8, 22.7, 19.8, 18.4, -5.3; FT-IR (thin film, $\left.\mathrm{cm}^{-1}\right)$ 2930, 2858, 1472, 1255, 1099, 836, 775; High-resolution $\mathrm{MS}\left(\mathrm{El}^{+}, \mathrm{m} / \mathrm{z}\right)$ molecular ion calcd for $\mathrm{C}_{15} \mathrm{H}_{28} \mathrm{O}_{1} \mathrm{Si}_{1} 252.1904$, found 252.1905, error $0.3 \mathrm{ppm}$.

Table 3, entry 6 . Solvent $=$ benzene; reaction temperature $=65{ }^{\circ} \mathrm{C} ; 10 \mathrm{~mol} \%$ complex 1; 9x0.25 mmol 1,5-COD (9 equiv). According to the general procedure ( $0.250 \mathrm{mmol}$ scale) to obtain $28 \mathrm{mg} 10 \mathrm{~F}$ as a colorless oil ( $49 \%$ yield). $\mathrm{R}_{f}=0.45$ (10\% ethyl acetate in hexanes). ${ }^{1} \mathrm{H}-\mathrm{NMR}\left(500 \mathrm{MHz}, \mathrm{CDCl}_{3}, \mathrm{ppm}\right) \delta 7.34-7.31$ (m, 4H), $7.28-7.24(\mathrm{~m}, 1 \mathrm{H}), 5.86(\mathrm{~s}, 1 \mathrm{H}), 5.60(\mathrm{~s}, 1 \mathrm{H}), 4.49(\mathrm{~s}, 2 \mathrm{H}), 4.05(\mathrm{~s}, 2 \mathrm{H})$, 2.20 (q, J = 7.5 Hz, 2H), 2.08 (br s, 4H), 1.03 (t, J = 7.5 Hz, 3H); ${ }^{13} \mathrm{C}-\mathrm{NMR}(125$ $\left.\mathrm{MHz}, \mathrm{CDCl}_{3}, \mathrm{ppm}\right) \delta 138.5,137.8,134.4,128.3,127.9,127.5,126.8,120.8,71.6$, 71.3, 24.5, 22.6, 22.3, 13.0; FT-IR (thin film, $\mathrm{cm}^{-1}$ ) 2933, 1454, 1356, 1067, 1028, 827, 734; High-resolution MS $\left(\mathrm{ESI}^{+}, \mathrm{m} / \mathrm{z}\right)$ molecular ion calcd for $\mathrm{C}_{16} \mathrm{H}_{20} \mathrm{O}_{1}$ 228.1514, found 251.1406, [M + Na] $]^{+}$, error $1.8 \mathrm{ppm}$.

Table 3, entry 7. Solvent $=$ benzene; reaction temperature $=65{ }^{\circ} \mathrm{C} ; 5 \mathrm{~mol} \%$ complex 1; $9 \times 0.25 \mathrm{mmol} 1,5-\mathrm{COD}$ (9 equiv). According to the general procedure $\left(0.250 \mathrm{mmol}\right.$ scale) to obtain $71 \mathrm{mg} \mathbf{1 0 G}$ as a pale yellow oil $(72 \%$ yield $) . \mathrm{R}_{f}=$ 0.42 (30\% ethyl acetate in hexanes). ${ }^{1} \mathrm{H}-\mathrm{NMR}\left(500 \mathrm{MHz}, \mathrm{CDCl}_{3}, \mathrm{ppm}\right) \delta 7.78(\mathrm{~d}$, $\mathrm{J}=8.5 \mathrm{~Hz}, 2 \mathrm{H}), 7.29(\mathrm{~d}, \mathrm{~J}=8.5 \mathrm{~Hz}, 2 \mathrm{H}), 5.69(\mathrm{~s}, 1 \mathrm{H}), 5.60(\mathrm{~s}, 1 \mathrm{H}), 3.85-3.81$ $(\mathrm{m}, 2 \mathrm{H}), 3.54(\mathrm{t}, \mathrm{J}=8.0 \mathrm{~Hz}, 2 \mathrm{H}), 2.43(\mathrm{~s}, 3 \mathrm{H}), 2.06-2.01(\mathrm{~m}, 4 \mathrm{H}), 1.88(\mathrm{~d}, \mathrm{~J}=$ $1.5 \mathrm{~Hz}, 3 \mathrm{H}), 1.35(\mathrm{~s}, 9 \mathrm{H}) ;{ }^{13} \mathrm{C}-\mathrm{NMR}\left(125 \mathrm{MHz}, \mathrm{CDCl}_{3}, \mathrm{ppm}\right) \delta 150.8,144.0,137.5$, 134.4, 132.6, 129.2, 127.7, 124.4, 122.9, 84.0, 47.5, 33.9, 27.8, 22.8, 22.5, 21.5, 19.5; FT-IR (thin film, $\mathrm{cm}^{-1}$ ) 2932, 1728, 1357, 1287, 1157, 1090, 977, 812; Highresolution MS $\left(\mathrm{ESI}^{+}, \mathrm{m} / \mathrm{z}\right)$ molecular ion calcd for $\mathrm{C}_{21} \mathrm{H}_{29} \mathrm{NO}_{4} \mathrm{~S} 391.1817$, found 414.1717, $[\mathrm{M}+\mathrm{Na}]^{+}$, error $1.9 \mathrm{ppm}$.

Table 3, entry 8 . Solvent $=$ benzene; reaction temperature $=65{ }^{\circ} \mathrm{C} ; 10 \mathrm{~mol} \%$ complex 1; $9 \times 0.25 \mathrm{mmol} 1,5-\mathrm{COD}$ (9 equiv). According to the general procedure $\left(0.250 \mathrm{mmol}\right.$ scale) to obtain $25 \mathrm{mg} \mathbf{1 0 H}$ as a colorless oil $(61 \%$ yield $) . \mathrm{R}_{f}=0.39$ (10\% ethyl acetate in hexanes). ${ }^{1} \mathrm{H}-\mathrm{NMR}\left(500 \mathrm{MHz}, \mathrm{CDCl}_{3}, \mathrm{ppm}\right) \delta 6.87$ (t, J = 
$10.0 \mathrm{~Hz}, 1 \mathrm{H}), 5.66(\mathrm{~d}, \mathrm{~J}=1.5 \mathrm{~Hz}, 1 \mathrm{H}), 4.21(\mathrm{q}, \mathrm{J}=7.0 \mathrm{~Hz}, 2 \mathrm{H}), 2.23-2.18(\mathrm{~m}$, 2H), $2.09-2.04(\mathrm{~m}, 2 \mathrm{H}), 1.95(\mathrm{~d}, \mathrm{~J}=1.5 \mathrm{~Hz}, 3 \mathrm{H}) 1.31(\mathrm{t}, \mathrm{J}=7.0 \mathrm{~Hz}, 3 \mathrm{H}) ;{ }^{13} \mathrm{C}-$ NMR $\left(125 \mathrm{MHz}, \mathrm{CDCl}_{3}, \mathrm{ppm}\right) \delta 166.9,137.4,132.2,130.1,124.3,60.3,23.0$, 21.5, 20.6, 14.2; FT-IR (thin film, $\mathrm{cm}^{-1}$ ) 2940, 1719, 1451, 1382, 1262, 1076; High-resolution MS (ESI+, $\mathrm{m} / \mathrm{z})$ molecular ion calcd for $\mathrm{C}_{10} \mathrm{H}_{14} \mathrm{O}_{2} 166.1059$, found 167.1067, $[\mathrm{M}+\mathrm{H}]^{+}$, error $0.2 \mathrm{ppm}$.

\section{N,O-cycloadduct 11:}

A mixture of cyclohexadiene $8 \mathrm{C}$ ( $45 \mathrm{mg}, 0.2 \mathrm{mmole}, 1.0$ equiv) and BocNHOH (32 mg, 0.24 mmole, 1.2 equiv) was dissolved in $4.0 \mathrm{~mL} \mathrm{CH}_{2} \mathrm{Cl}_{2}$ and this mixture was cooled to $0{ }^{\circ} \mathrm{C}$ using an ice bath. Tetrapropyl ammonium periodate (90 mg, 0.24 mmole, 1.2 equiv) was dissolved in $2.0 \mathrm{~mL} \mathrm{CH}_{2} \mathrm{Cl}_{2}$ and this solution was taken up in a gas tight syringe. This solution of the oxidant was added to the solution of the cyclohexadiene and the hydroxamic acid in $\mathrm{CH}_{2} \mathrm{Cl}_{2}$ maintained at $0{ }^{\circ} \mathrm{C}$ over $2 \mathrm{~h}$ using syringe pump. The analysis of the crude reaction mixture revealed complete consumption of the cyclohexadiene. The reaction mixture was concentrated and purified by flash column chromatography using silica gel. Gradient elution with $10 \%$ ethyl acetate in hexanes $(100 \mathrm{~mL})$ followed by $20 \%$ ethyl acetate in hexanes afforded the desired hetero-diels alder adduct 11 (53 mg, $75 \%$ yield) as a yellow oil. $\mathrm{R}_{f} 0.30$ (30\% ethyl acetate in hexanes). ${ }^{1} \mathrm{H}-\mathrm{NMR}\left(500 \mathrm{MHz}, \mathrm{CDCl}_{3}, \mathrm{ppm}\right) \delta 4.91(\mathrm{~d}, \mathrm{~J}=13.0 \mathrm{~Hz}, 1 \mathrm{H}), 4.79-$ $4.72(\mathrm{~m}, 4 \mathrm{H}), 4.70(\mathrm{~d}, \mathrm{~J}=13.0 \mathrm{~Hz}, 1 \mathrm{H}), 2.20-2.14(\mathrm{~m}, 1 \mathrm{H}), 2.12-2.08(\mathrm{~m}, 1 \mathrm{H})$, $2.06(\mathrm{~s}, 3 \mathrm{H}), 2.03(\mathrm{~s}, 3 \mathrm{H}), 1.49-1.43(\mathrm{~m}, 10 \mathrm{H}), 1.36-1.30(\mathrm{~m}, 1 \mathrm{H}) ;{ }^{13} \mathrm{C}-\mathrm{NMR}$ (125 MHz, $\left.\mathrm{CDCl}_{3}, \mathrm{ppm}\right) \delta 170.5,170.46,157.3,136.7,134.6,81.9,72.9,60.7$, 59.7, 52.1, 28.1, 24.0, 21.1. 20.74, 20.72; FT-IR (thin film, $\mathrm{cm}^{-1}$ ) 2939, 1742, 1701, 1456, 1370, 1225, 1164, 1026; High-resolution MS (ESI $\left.{ }^{+} \mathrm{m} / \mathrm{z}\right)$ molecular ion calcd for $\mathrm{C}_{17} \mathrm{H}_{25} \mathrm{NO}_{7} 355.1631$, found 378.1523 , [M+Na] $]^{+}$, error $0.9 \mathrm{ppm}$.

\section{Cycloadduct 12:}

To a solution of $8 \mathbf{A}(45.5 \mathrm{mg}, 0.203 \mathrm{mmol})$ in toluene $(2.0 \mathrm{~mL})$ was added maleic anhydride $(24.5 \mathrm{mg}, 0.250 \mathrm{mmol})$. This solution was heated to $105{ }^{\circ} \mathrm{C}$ for $12 \mathrm{~h}$. After concentration and in vacuo (rotary evaporator) the residue was further purified by column chromatography on silica gel, eluting with 1:1 ethyl acetate-hexanes to give $55.9 \mathrm{mg}$ of $\mathbf{1 2}\left(85 \%\right.$ yield). $R_{f}=0.26$ (1:1 ethyl acetatehexanes). ${ }^{1} \mathrm{H}$ NMR $\left(500 \mathrm{MHz}, \mathrm{CDCl}_{3}\right) \delta 5.95$ (dd, $\left.\mathrm{J}=1.5,6.0 \mathrm{~Hz}, 1 \mathrm{H}\right), 3.73(\mathrm{~s}$, $3 \mathrm{H}), 3.71(\mathrm{~s}, 3 \mathrm{H}), 3.57(\mathrm{t}, \mathrm{J}=7.5 \mathrm{~Hz}, 1 \mathrm{H}), 3.17(\mathrm{~m}, 2 \mathrm{H}), 3.10(\mathrm{~m}, 2 \mathrm{H}), 2.70(\mathrm{~m}$, $2 \mathrm{H}), 1.59(\mathrm{~m}, 2 \mathrm{H}), 1.37(\mathrm{~m}, 2 \mathrm{H}) ;{ }^{13} \mathrm{C}$ NMR $\left(125 \mathrm{MHz}, \mathrm{CDCl}_{3}\right) \delta$ 172.6, 172.4, 168.9, 168.8, 142.3, 126.2, 52.62. 52.60, 49.5, 44.8, 44.7, 36.1, 33.6, 32.1, 23.7, 22.8; FT-IR (thin film, $\mathrm{cm}^{-1}$ ) 2956, 2881, 1841, 1782, 1743, 1440, 1342, 1289, $1234,1158,1086,951,911$; high resolution ESI molecular ion calcd for 345.0945 $\mathrm{C}_{16} \mathrm{H}_{18} \mathrm{O}_{7} \mathrm{Na}$ found 345.0945 . 


\section{${ }^{1} \mathrm{H}$ and ${ }^{13} \mathrm{C}$ NMR Spectra}




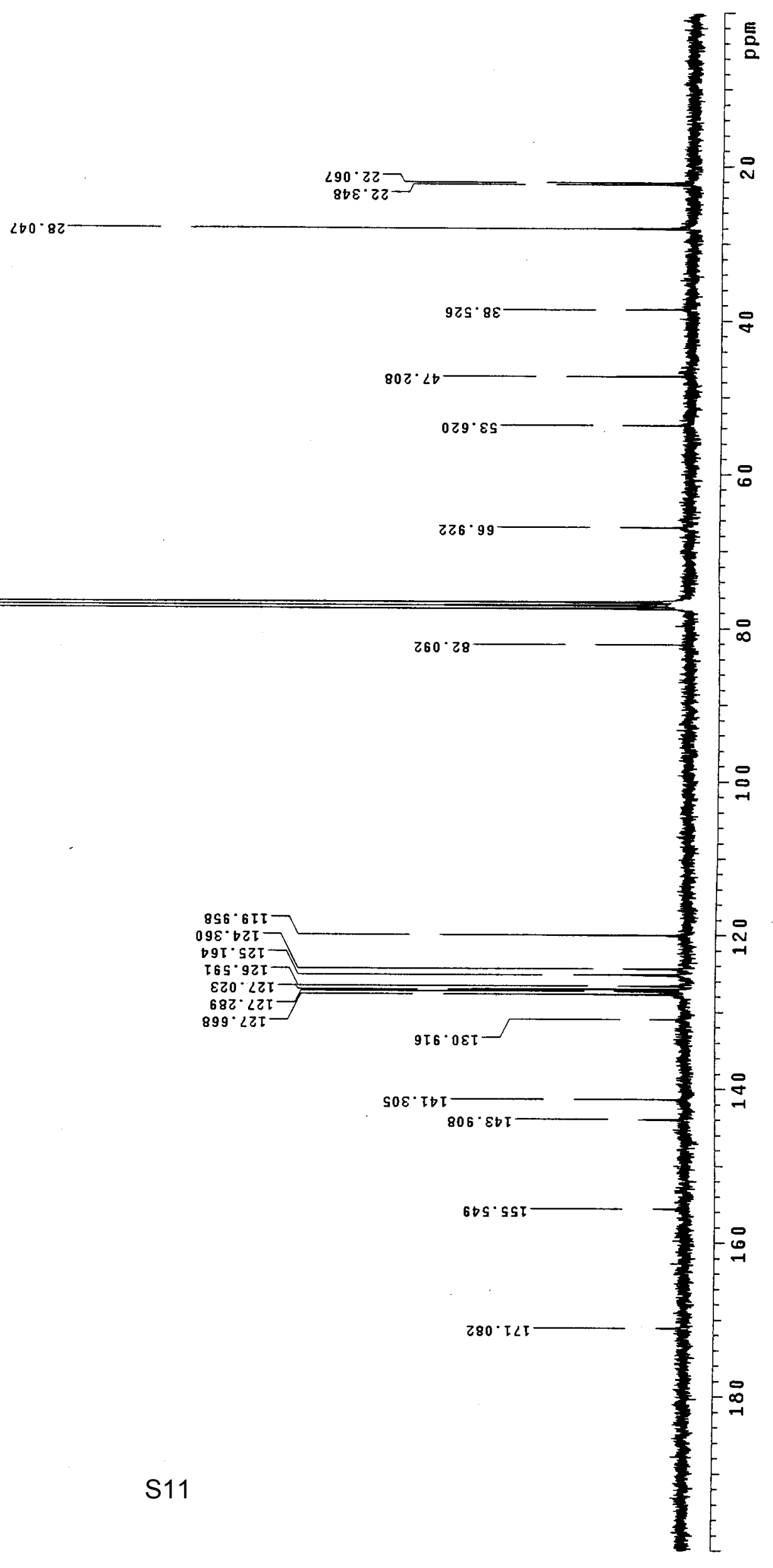




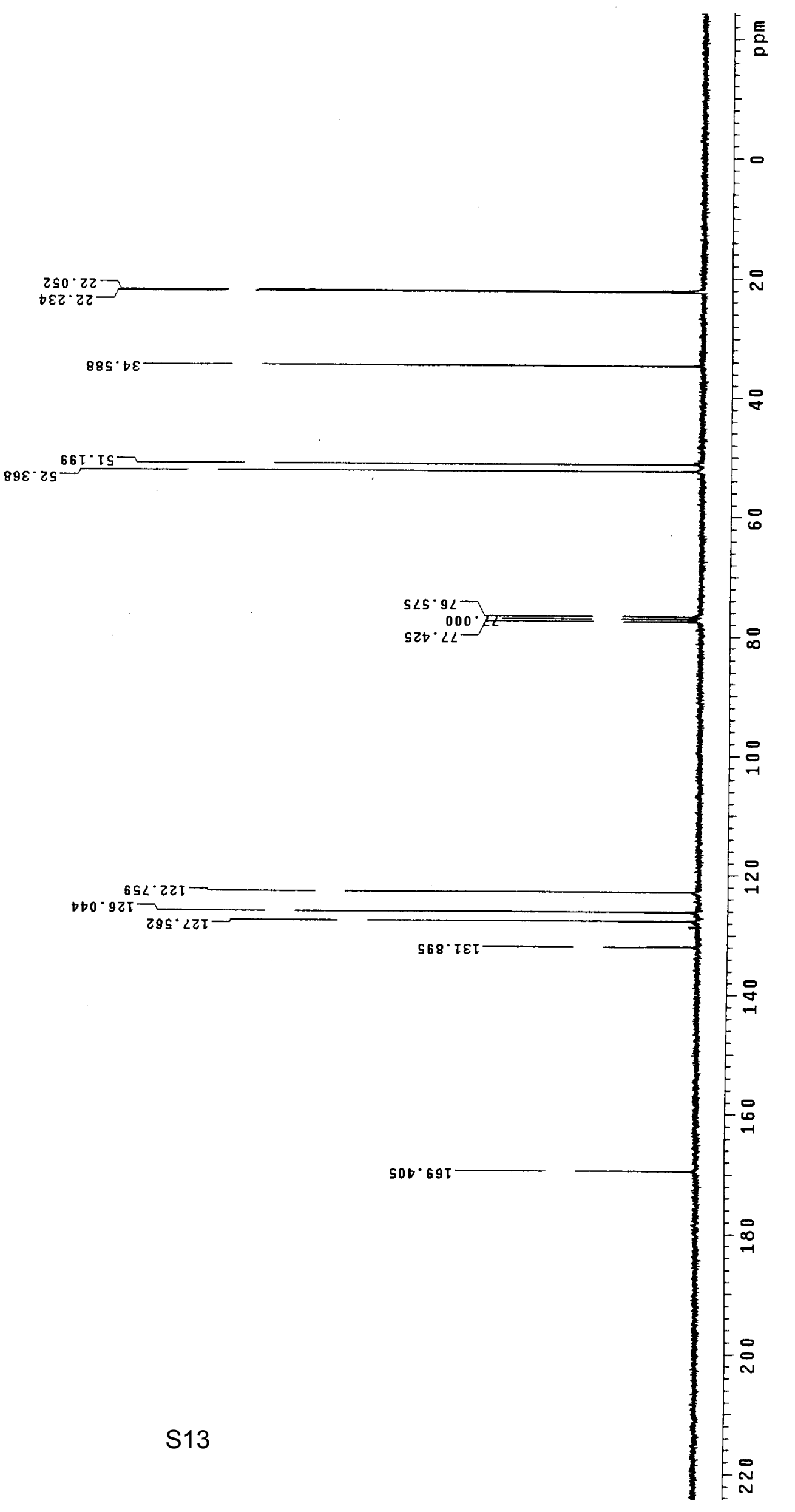




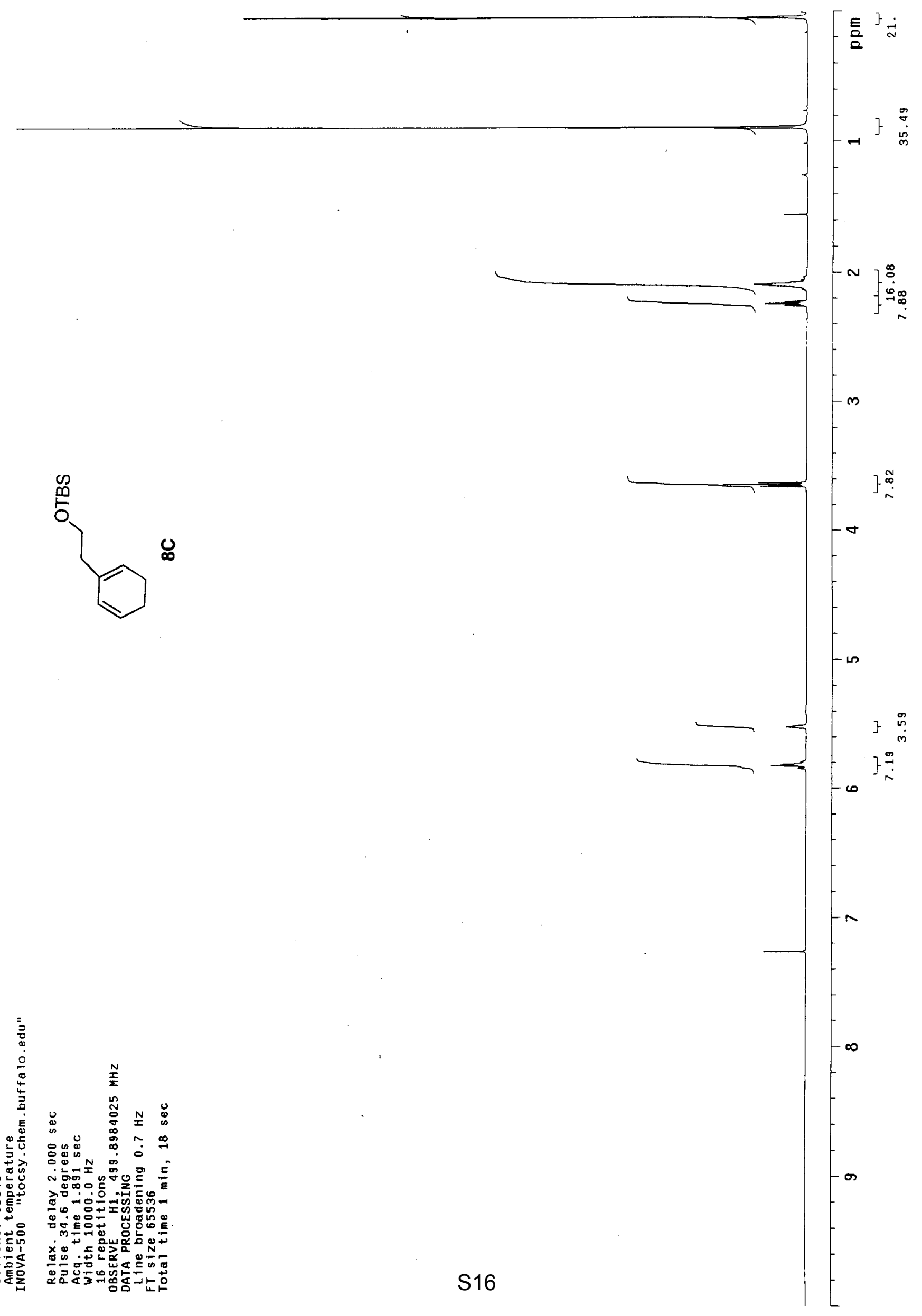



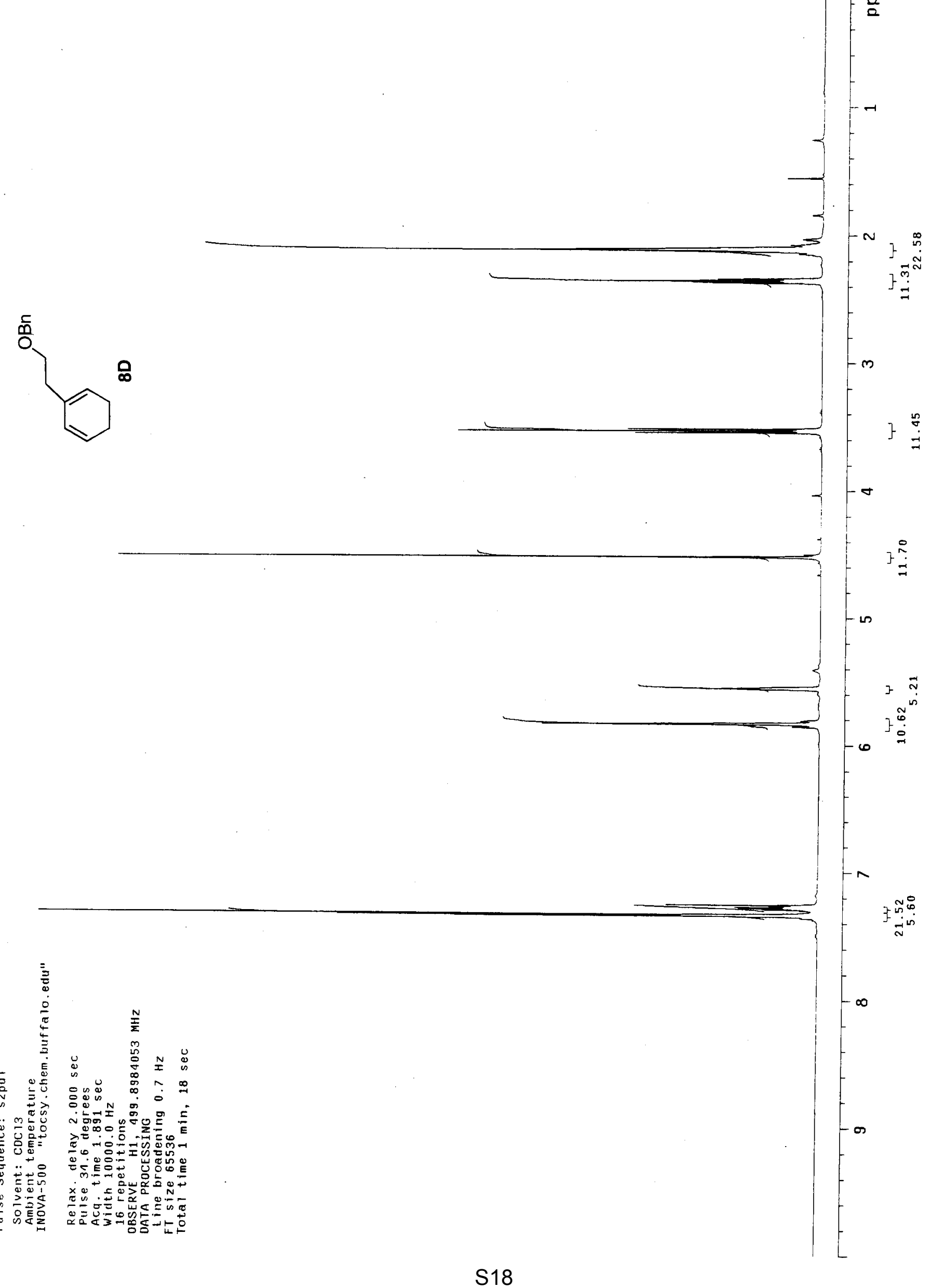
$966.5 \varepsilon-$

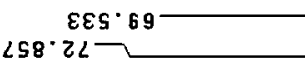<smiles>[O-]CCC1=CCCC=C1</smiles>

$\angle 58^{\circ} 22$ 


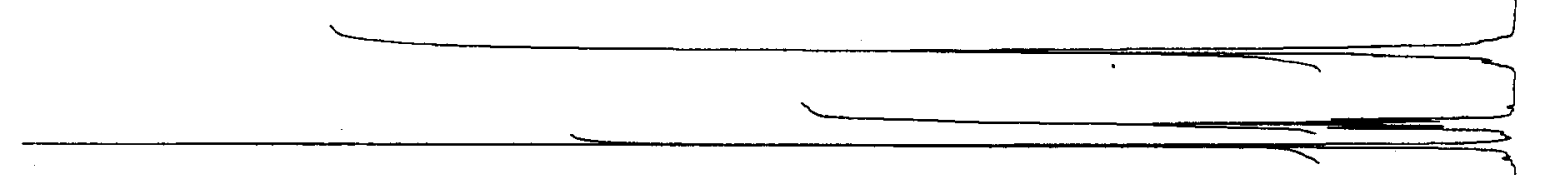<smiles>[O]CCC1=CCCC=C1</smiles>
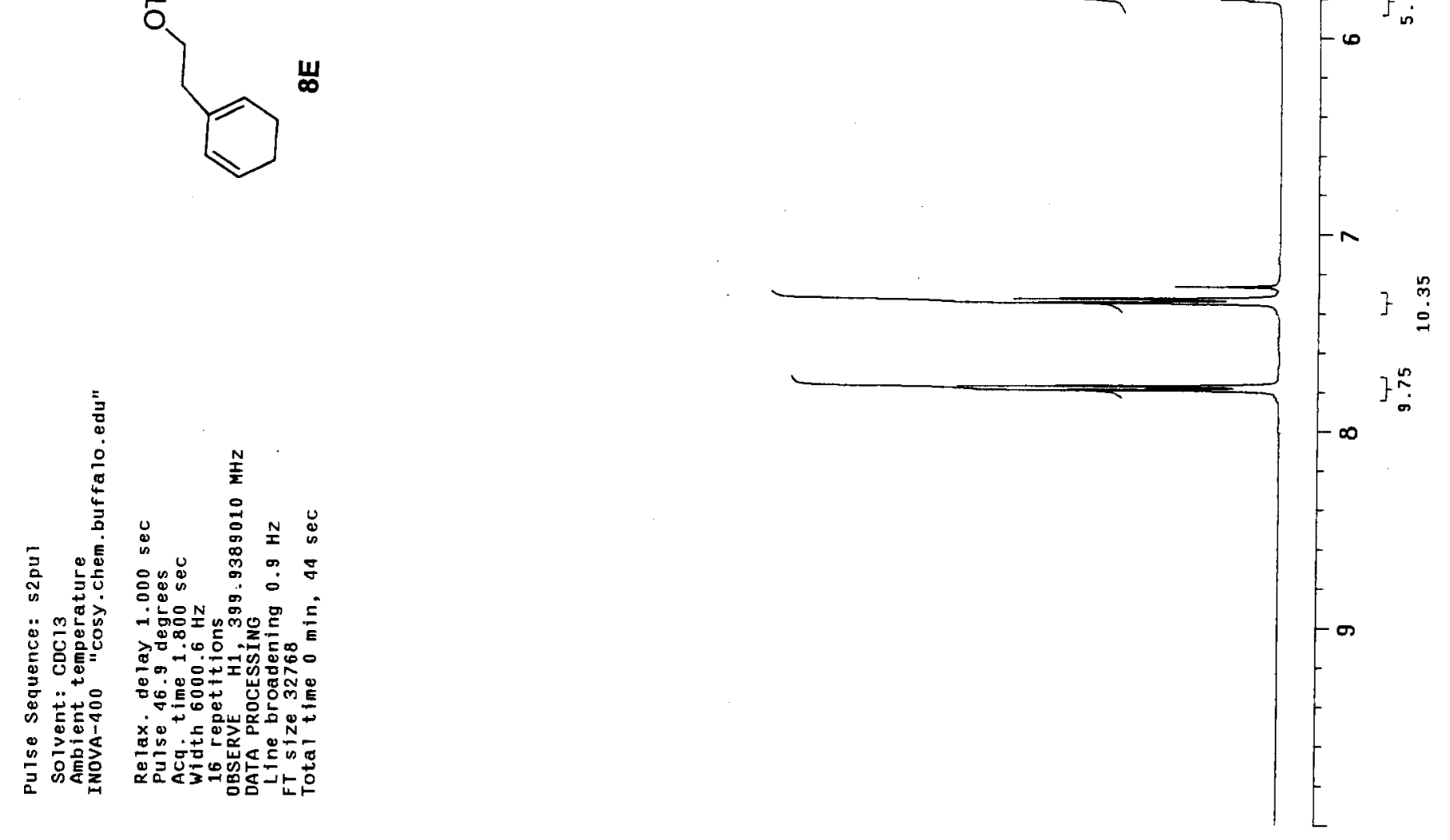


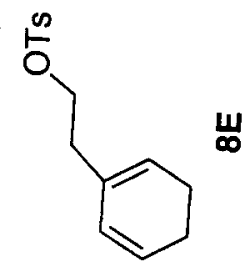

$\varepsilon S 8^{\circ} \bullet \varepsilon$

8SS'TZ

St $6^{\circ} \mathrm{I}$

SoE.69-

SLS. 92

$000 \cdot 32$

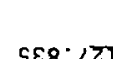

$\varepsilon \tau \varepsilon \cdot \varepsilon \tau \tau \longrightarrow$

$250 \cdot 92 \tau$

$0+\angle Z T$
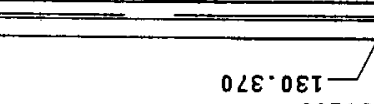

S\&T. $\varepsilon \varepsilon \tau$

TGS'bDI

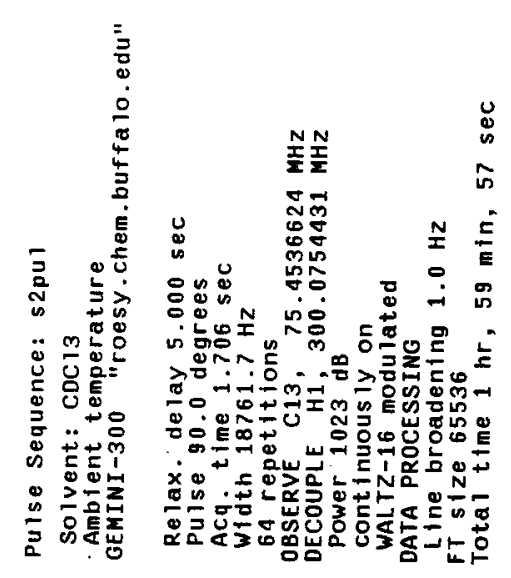




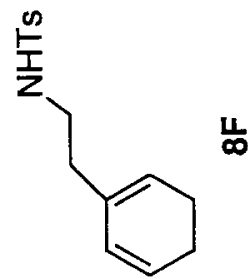

$\frac{u}{\infty}$
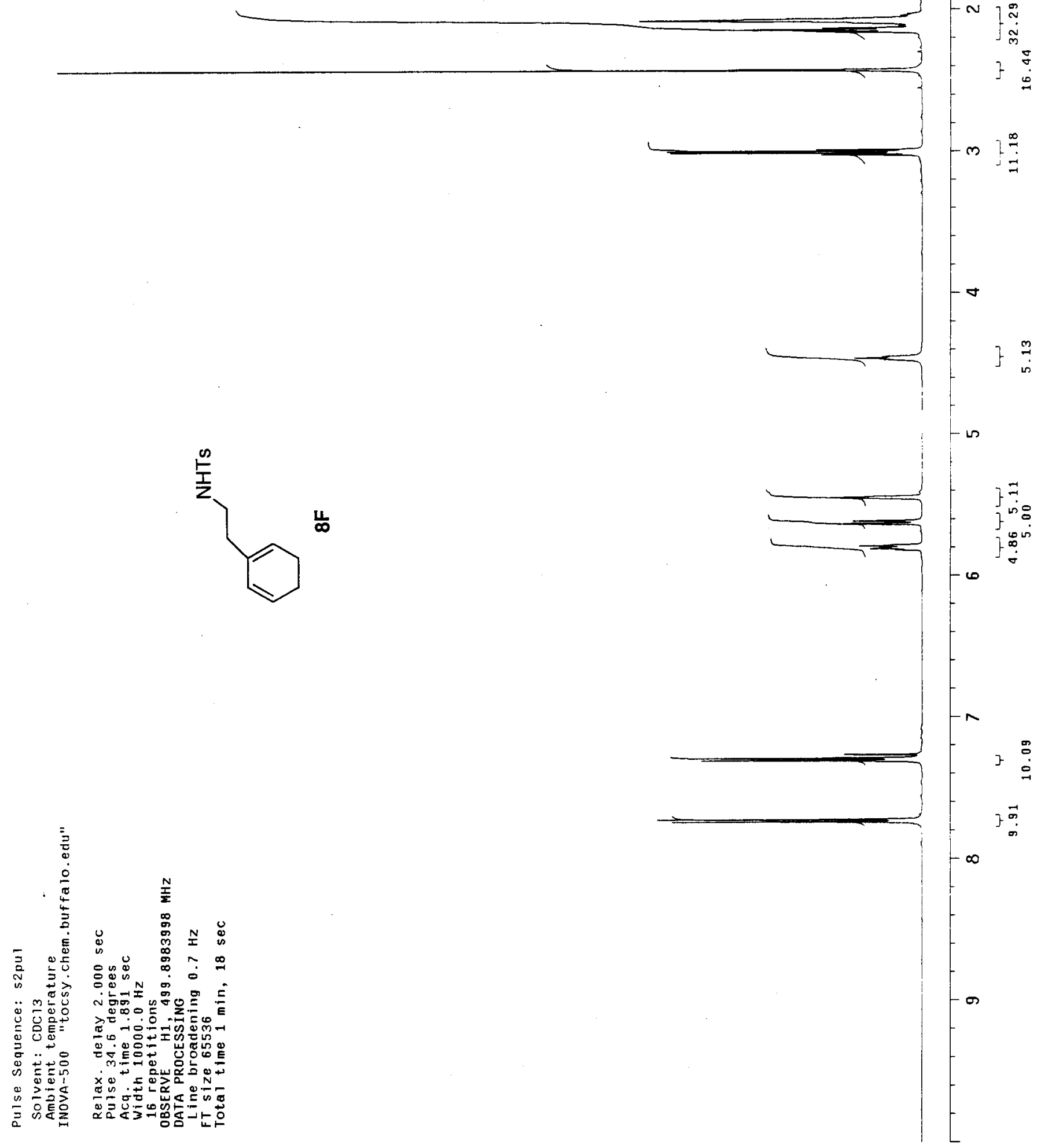

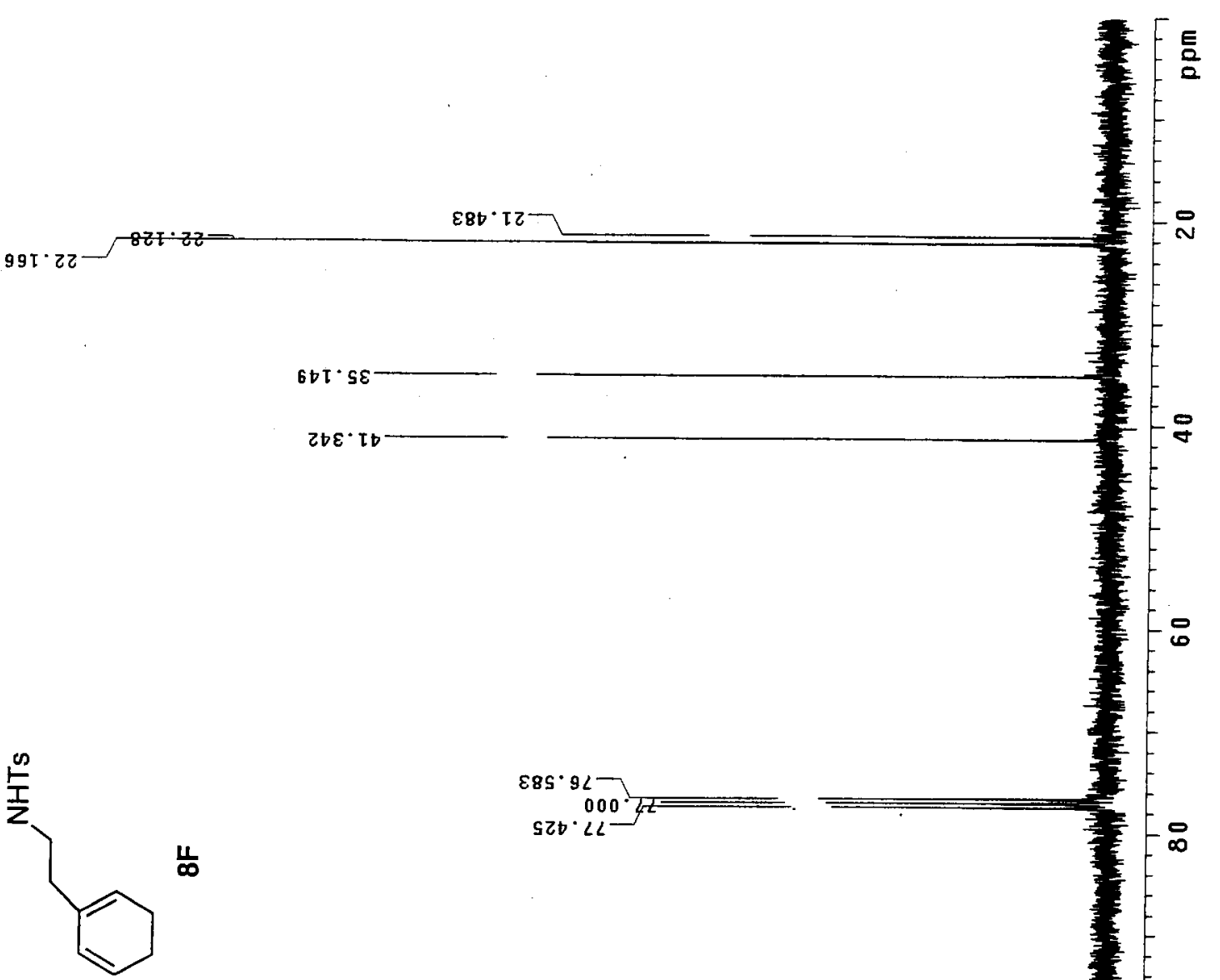

$\varepsilon 85 \cdot 9 L$

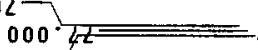

$\frac{11}{\infty}$

$s Z b \cdot \angle L$

$660^{\circ} \angle Z I$

$\varepsilon\llcorner\varepsilon \cdot \varepsilon 己 \tau$

$\angle 29 \cdot 5 z 1-$

$520.82 \mathrm{I}$

$609.62 \tau$

$289^{\circ} \sin$

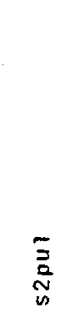

$=$

告

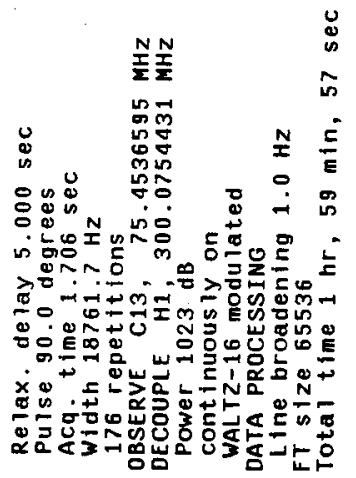

$9 \tau \varepsilon^{\cdot} \varepsilon \sqcup \tau$

$\ddot{0}$ mㄴㅇㄴ

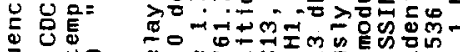

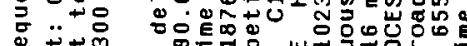

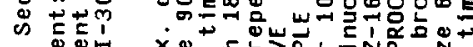

\& 2 然

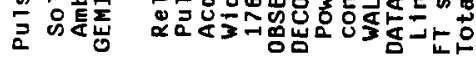



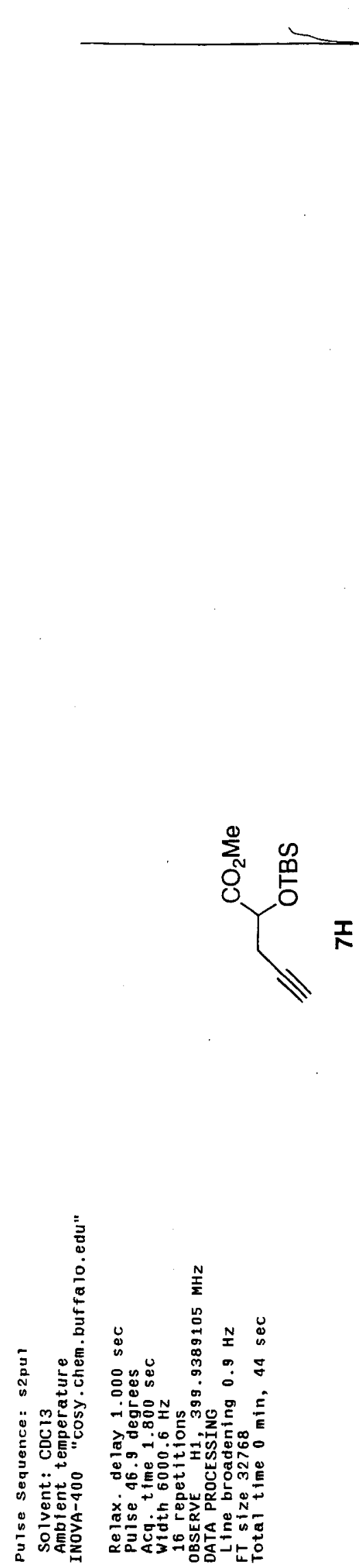


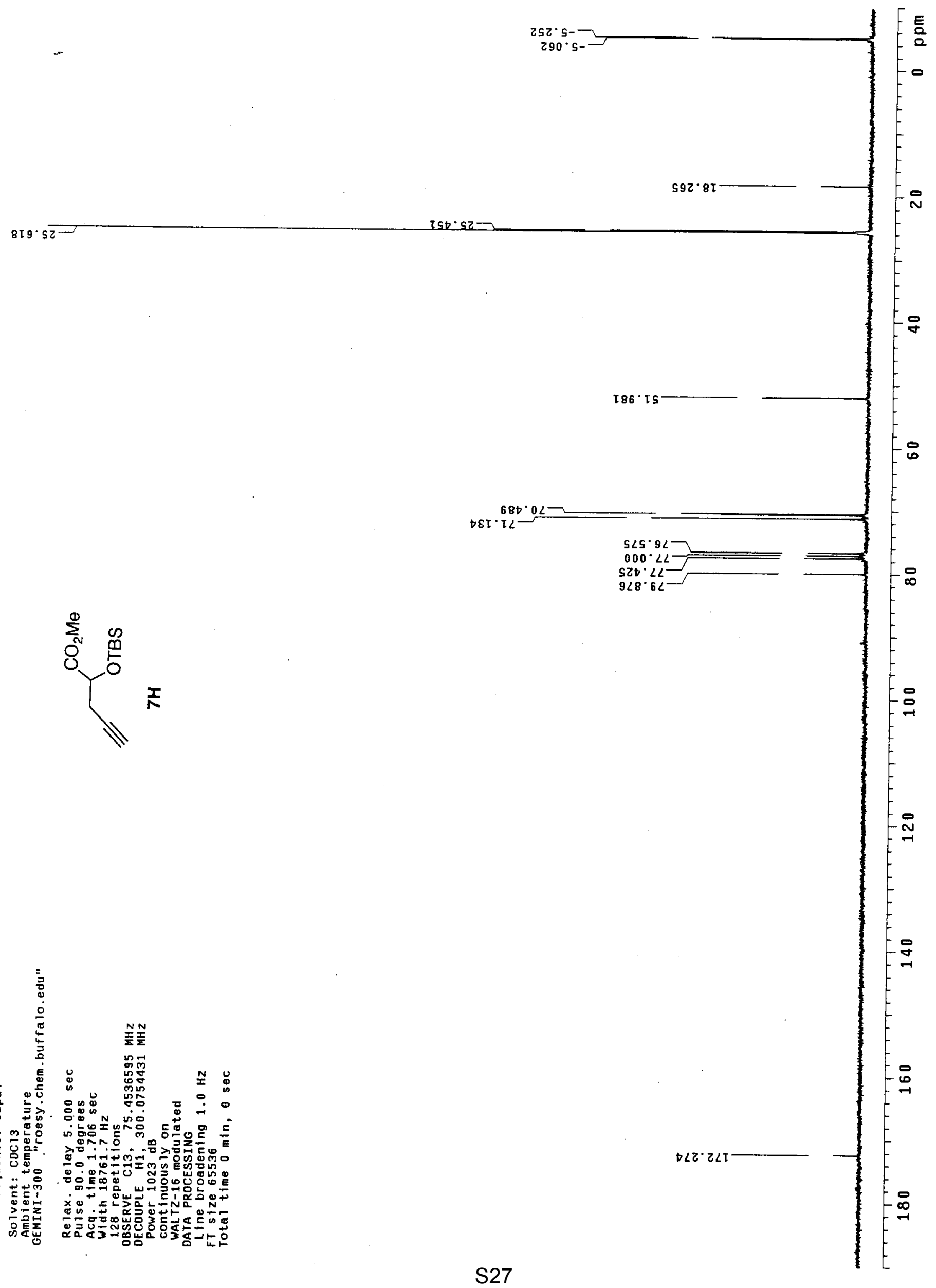



a
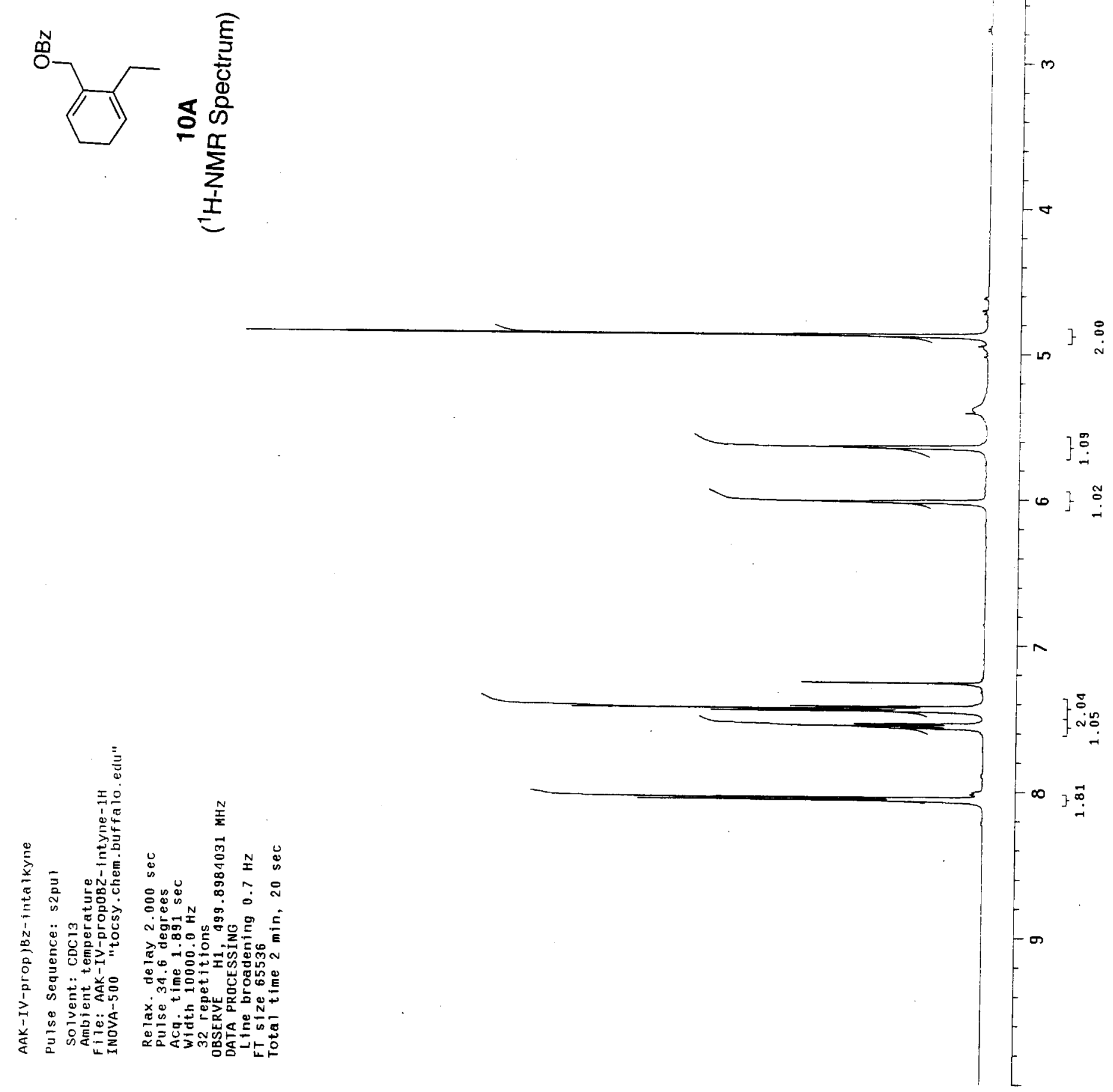

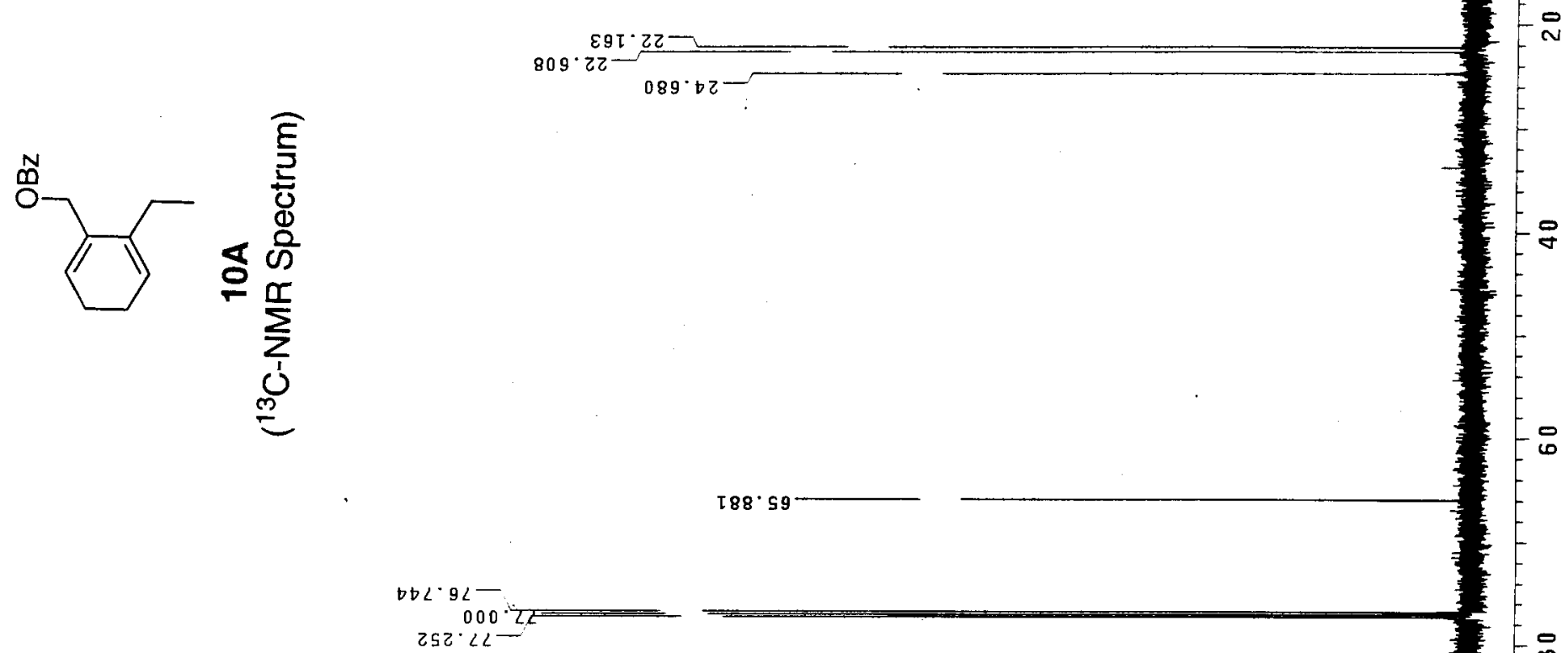

$\tau 88^{\circ} 59^{\circ}$
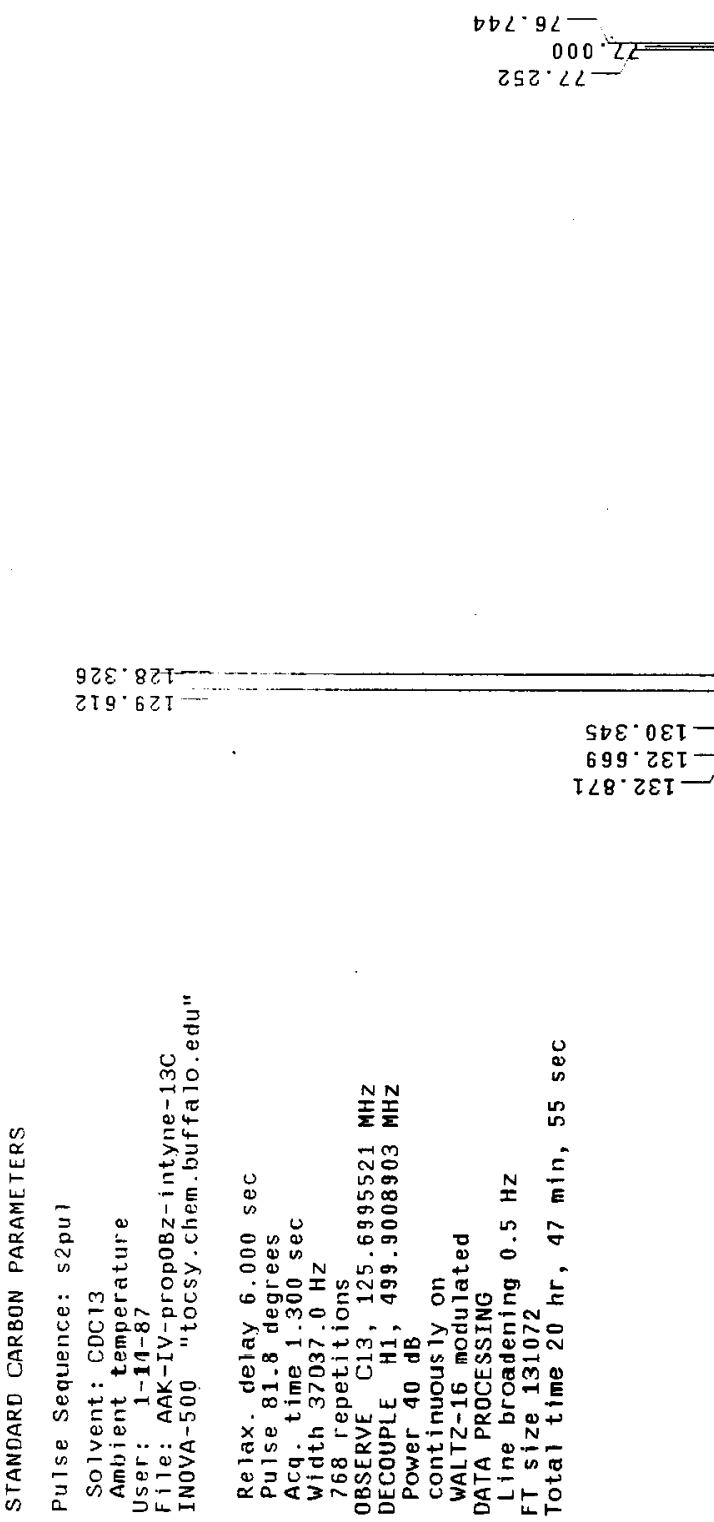

0 II 821

$\angle 8^{\circ} 2 \varepsilon \tau$ 


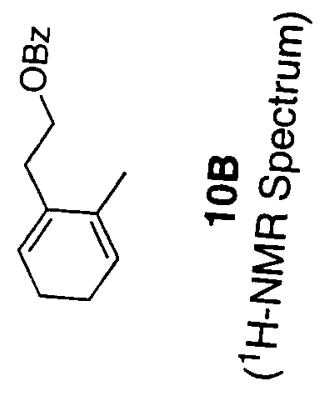
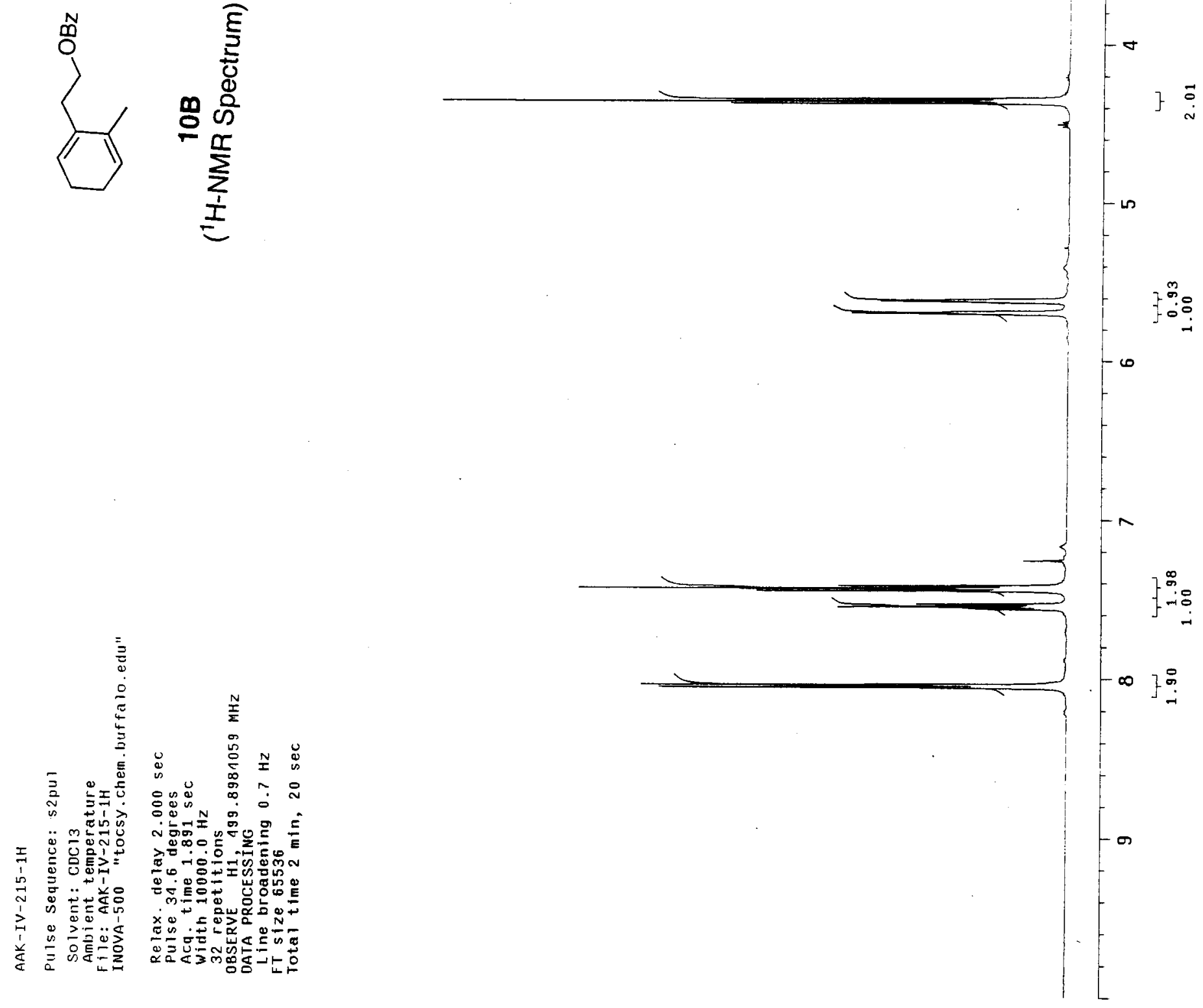


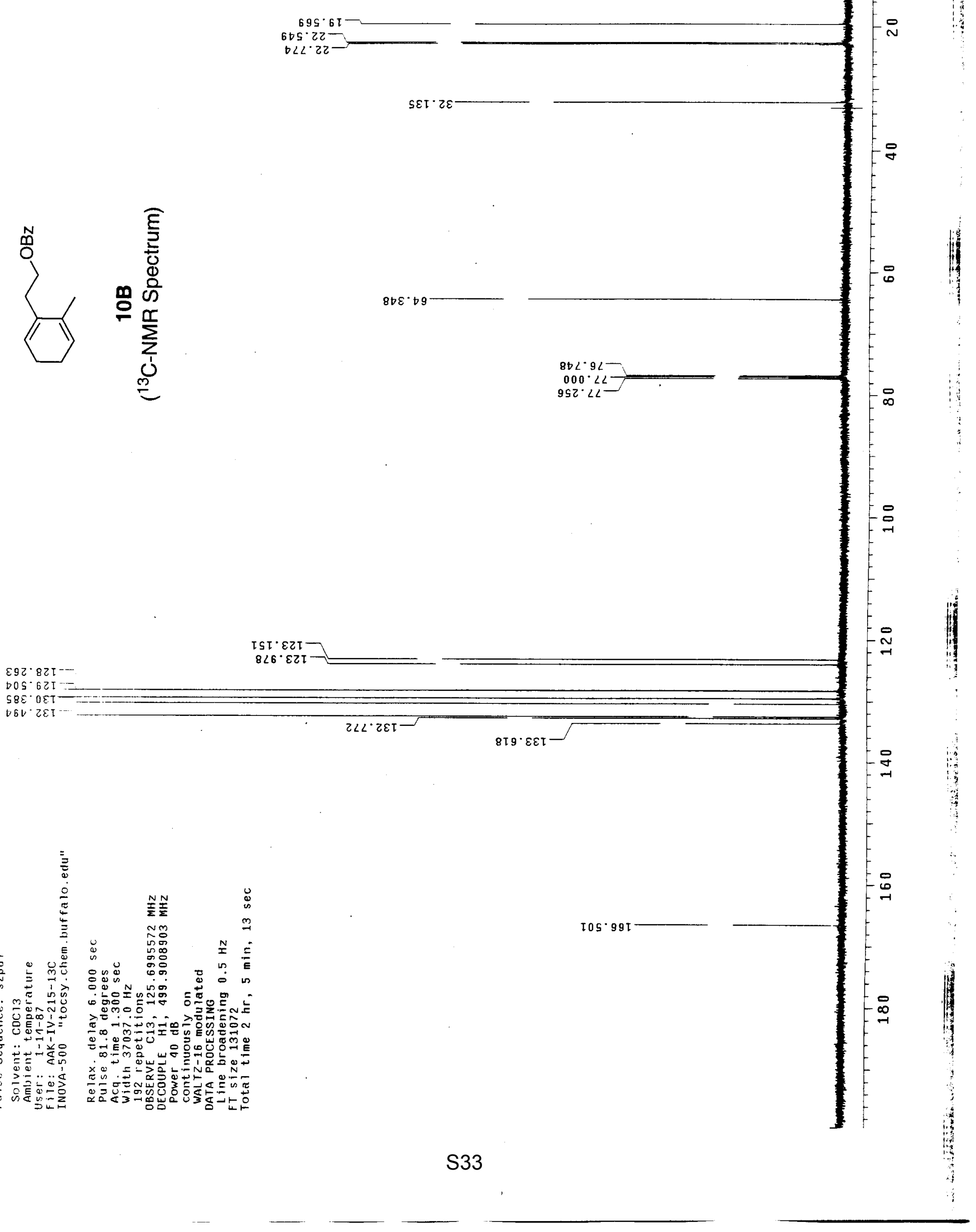


我
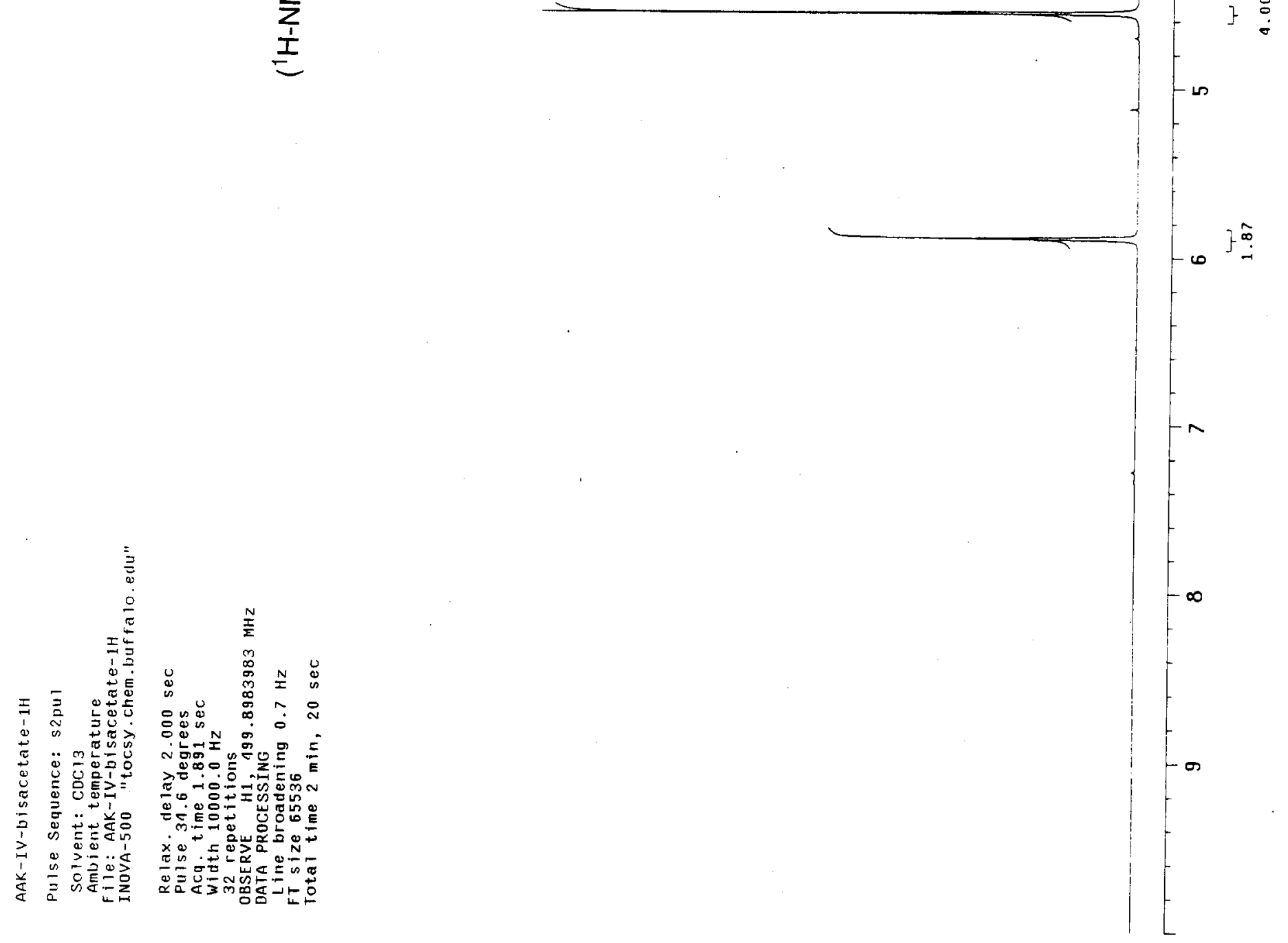


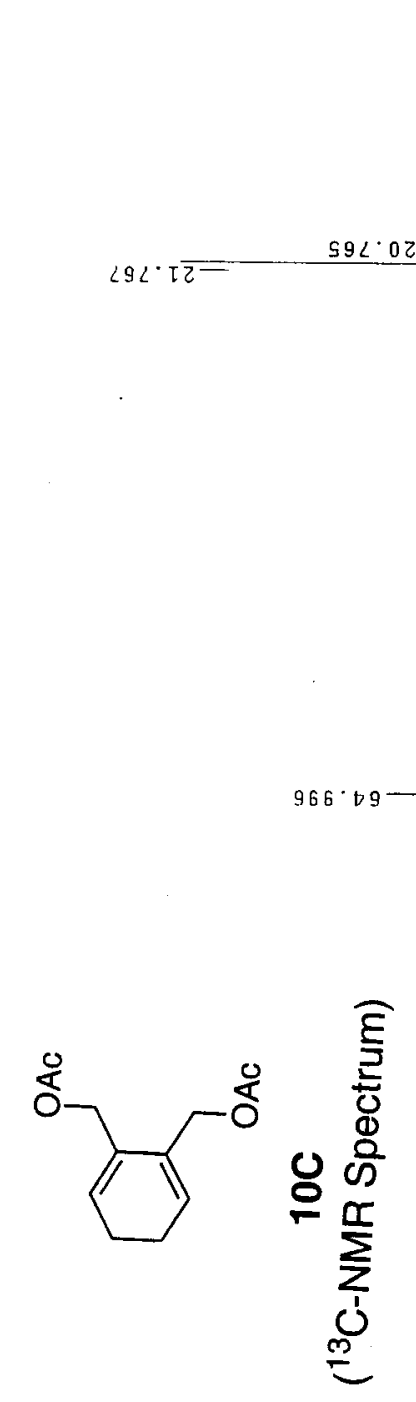

$0 \angle 8 \cdot 821^{\cdots} \ldots \ldots$

$\nabla \varepsilon \nabla \cdot 0 \varepsilon \tau$
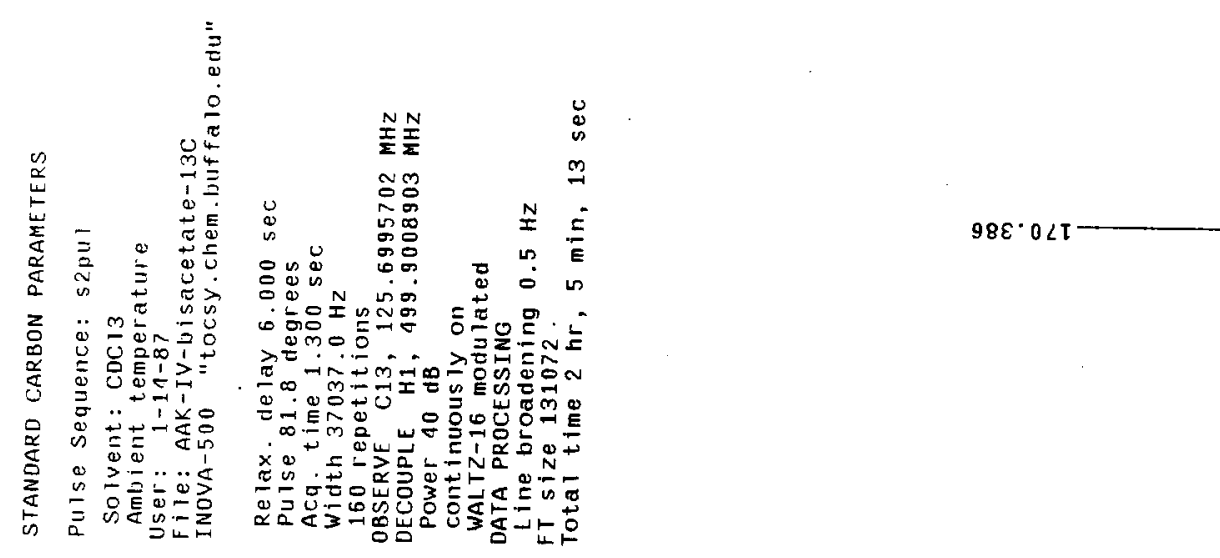


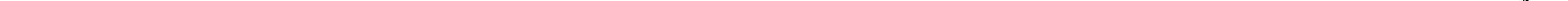



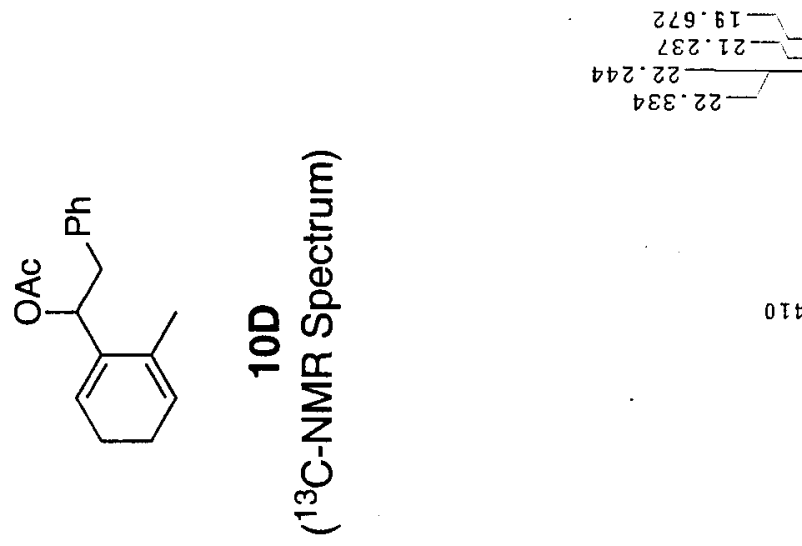

$802.92-$

$6 \varepsilon z \cdot \circ<-$

000
$9 S Z$$\angle L$

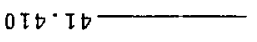

G
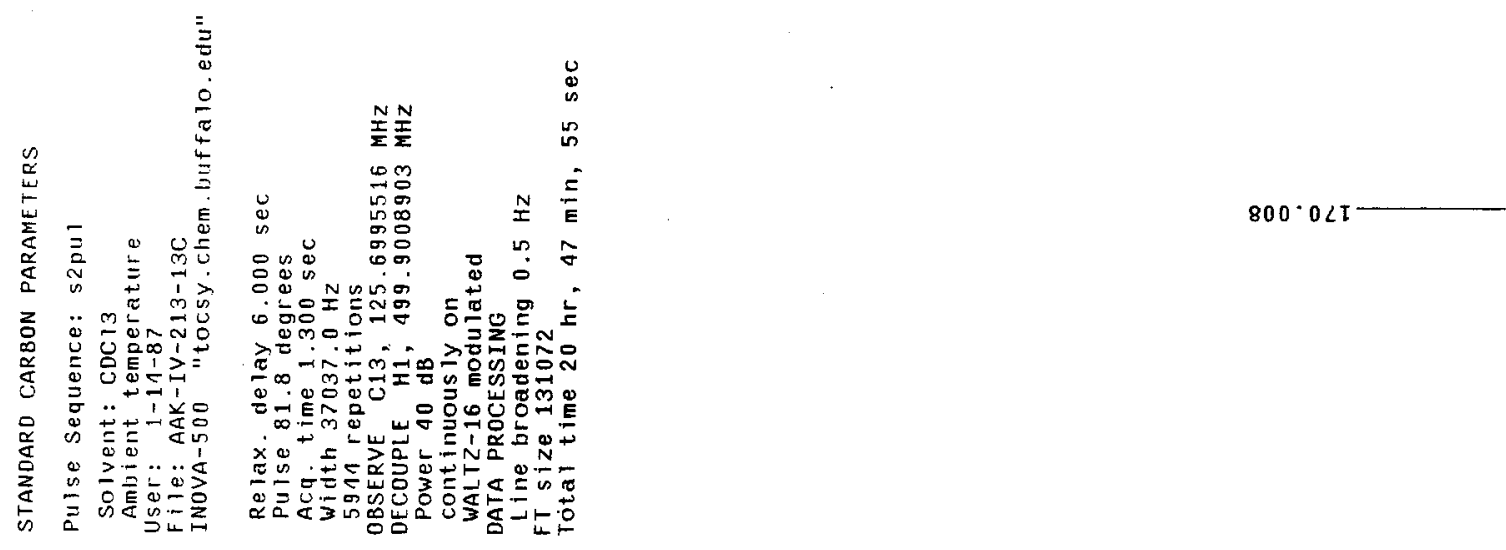


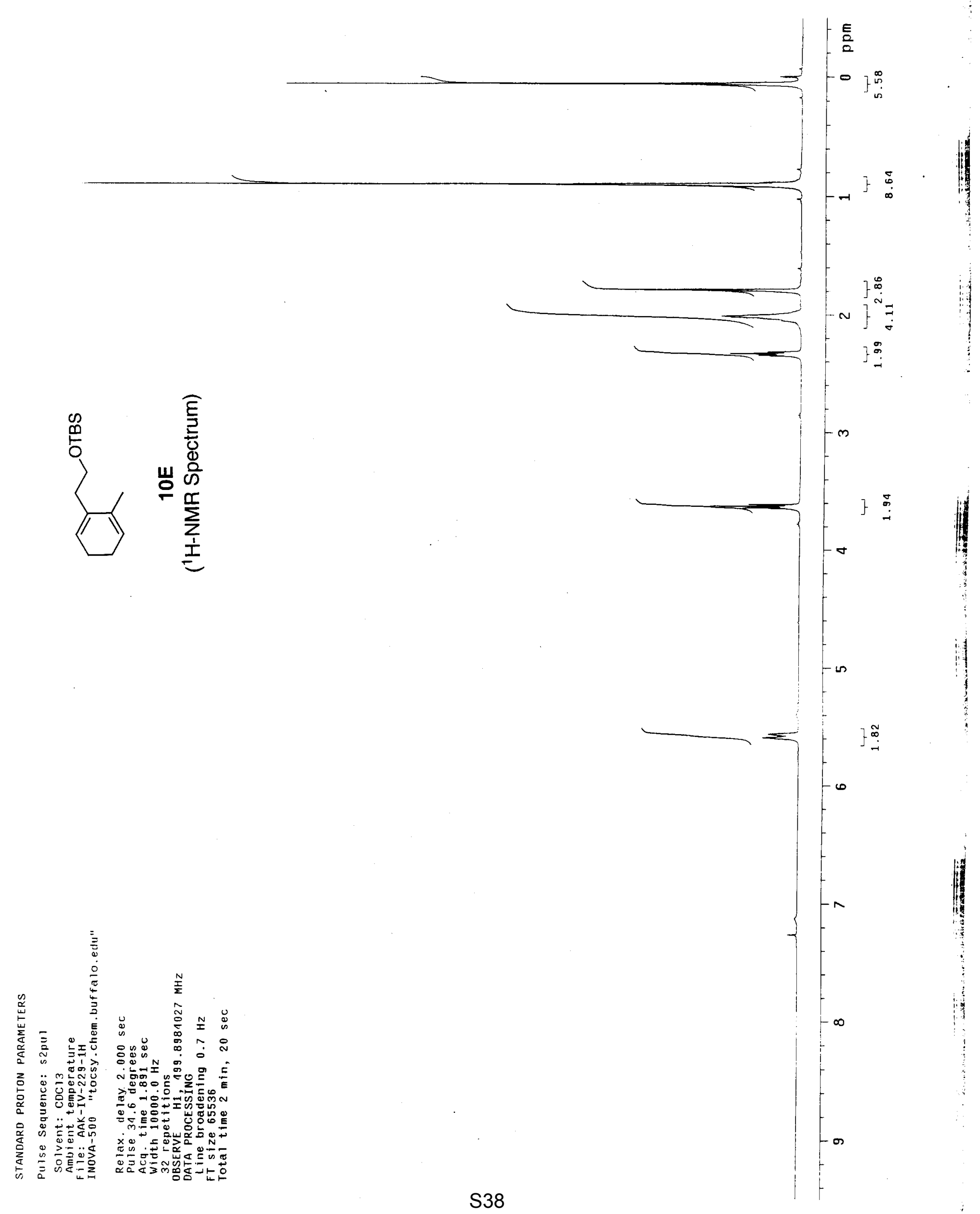


โ82.5-

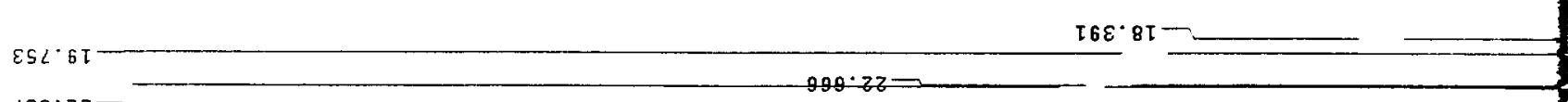

ㅇ

$2 \varepsilon 8 \cdot 2 z=$

-

$60 s^{\circ} 9 \varepsilon$

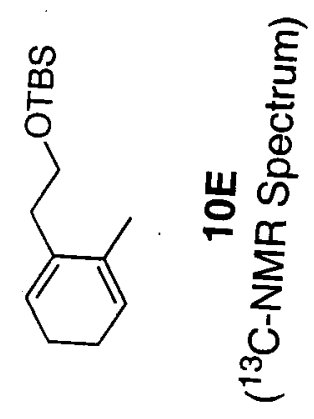

$029^{\circ} \varepsilon 9$

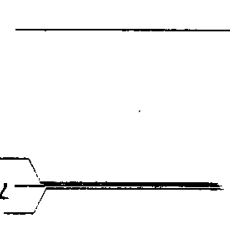

00292
$000.2 L$

252.24

$9 \operatorname{si}^{\circ} \varepsilon \varepsilon I$

$9 \tau 9^{\circ} \sqcup \mathcal{E}$

$\stackrel{8}{-1}$

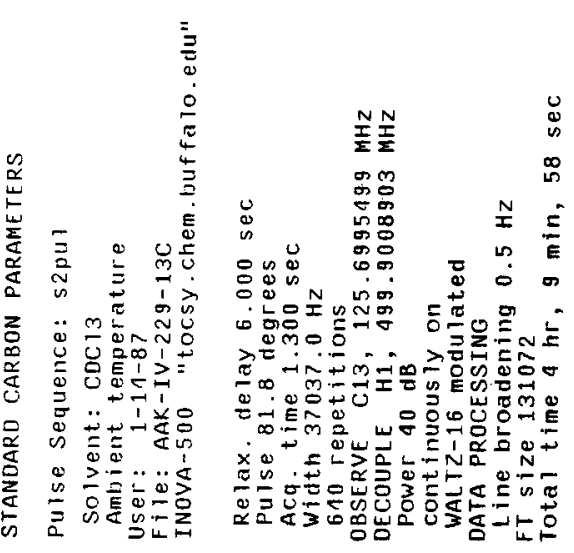




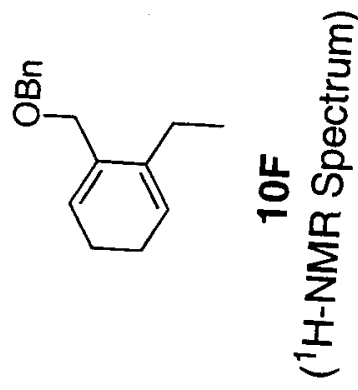

[

$-7 \underset{\sim}{\stackrel{\infty}{\infty}}$

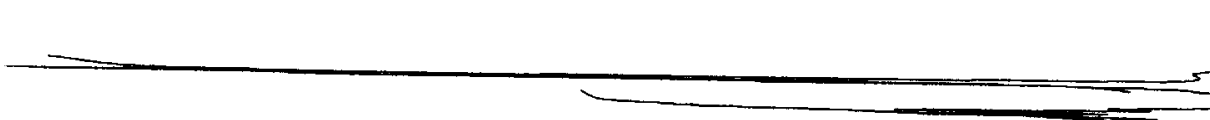

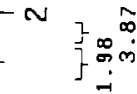

$m$

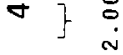

I

- in

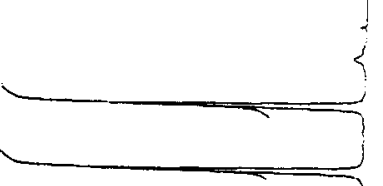

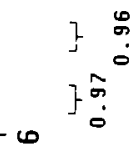

0
0
0
0
0
0
0
0

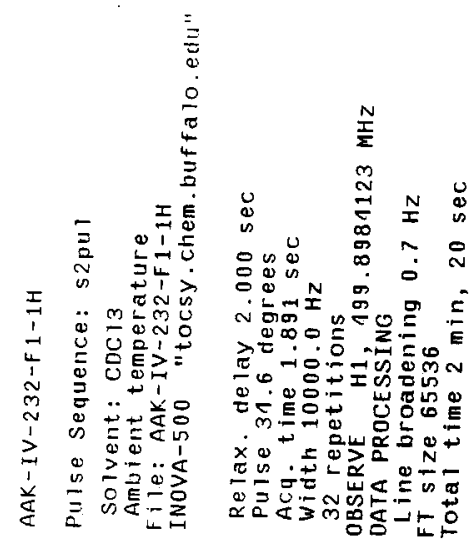



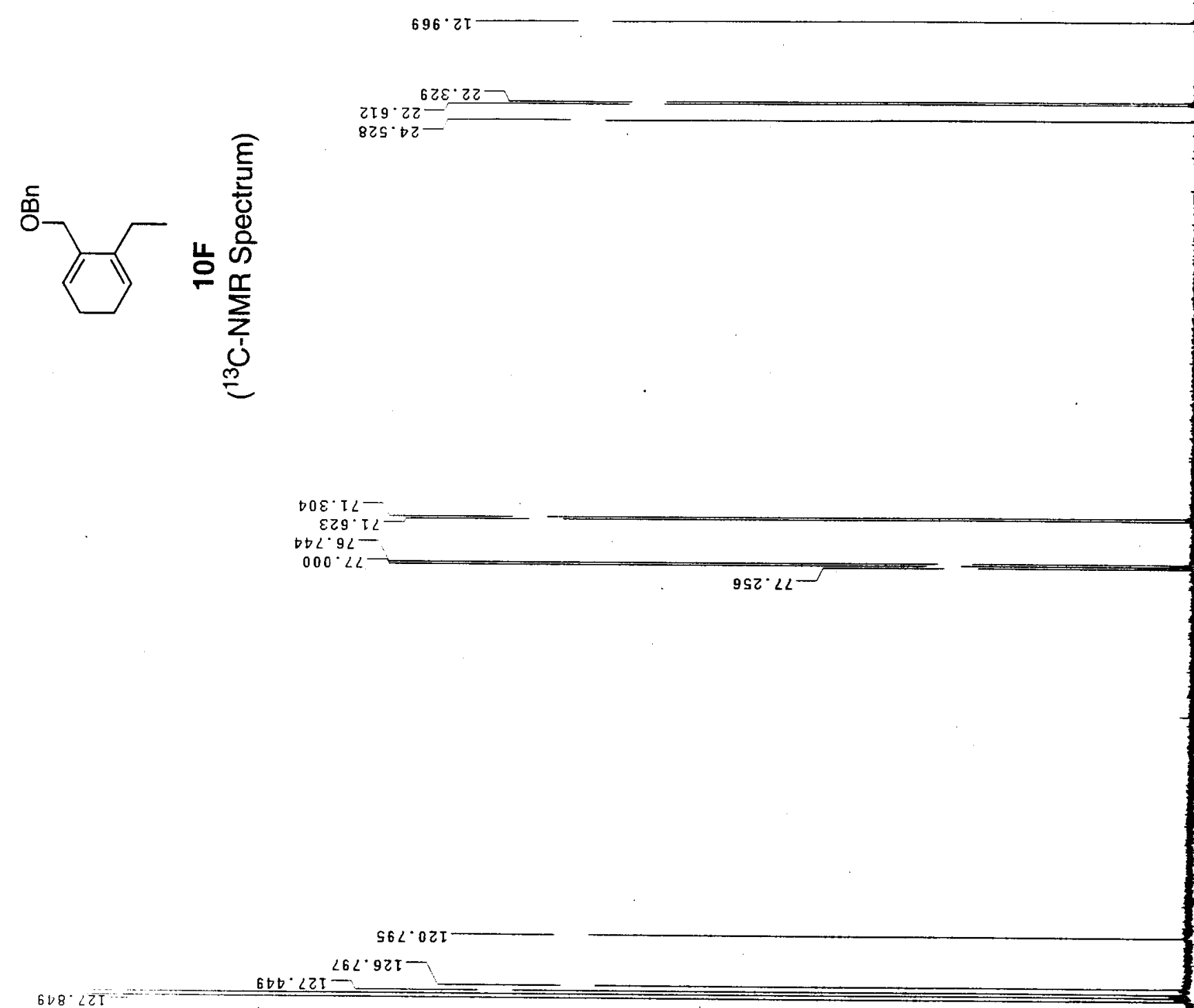

$628 \cdot \angle Z I-$
$Z \angle Z \cdot 8 Z 1-$

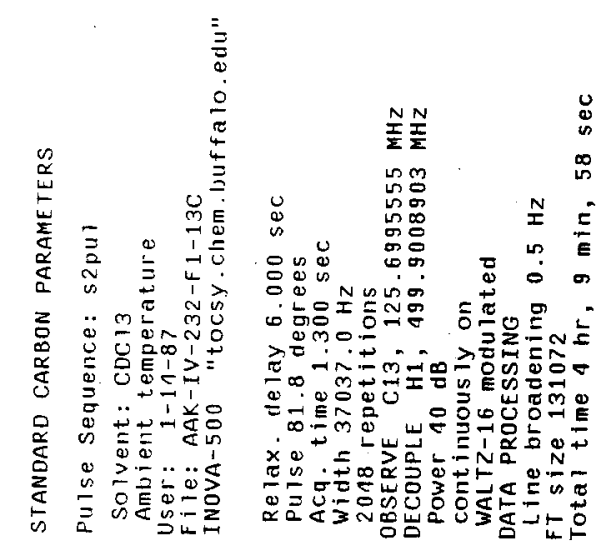




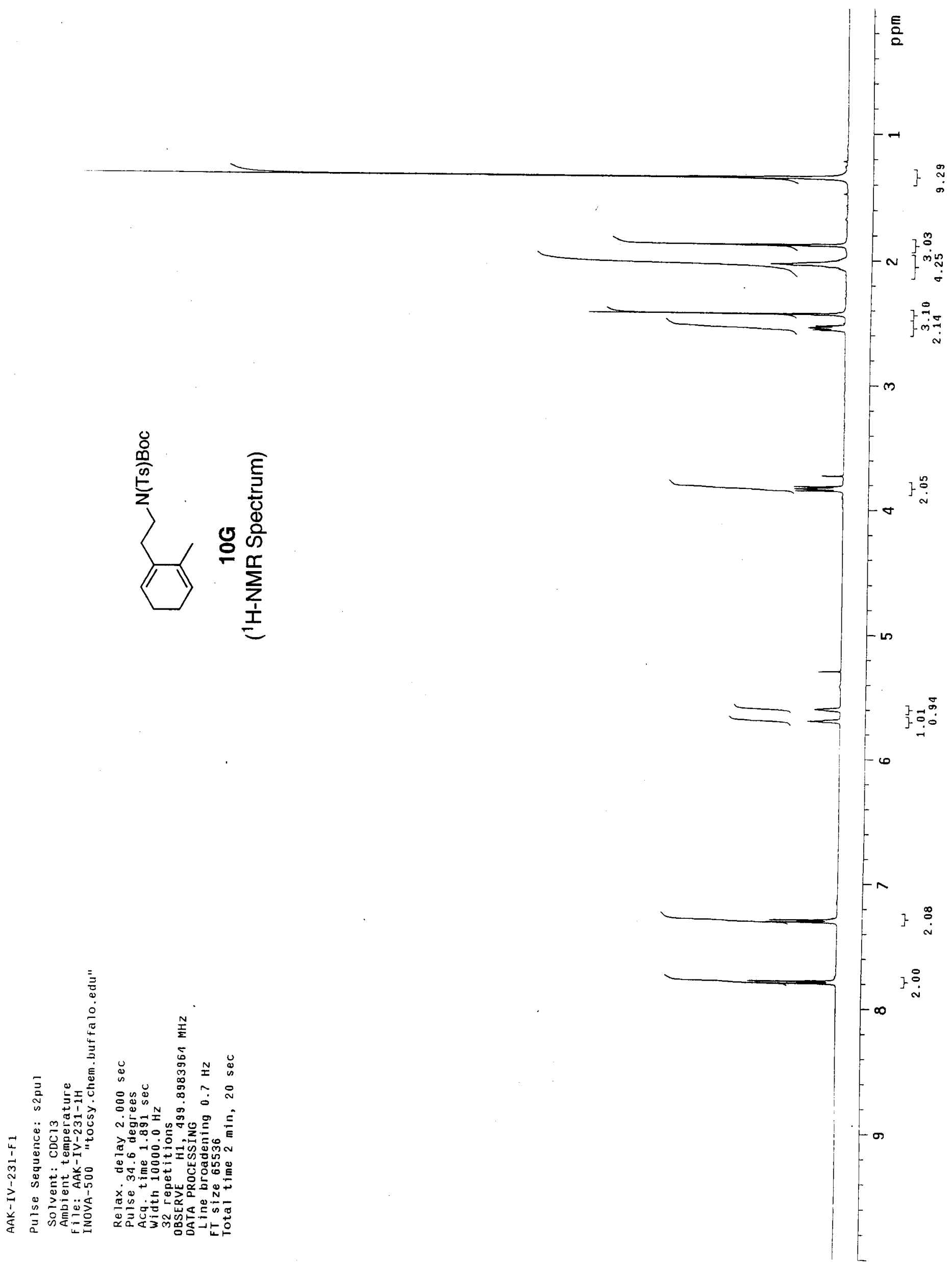




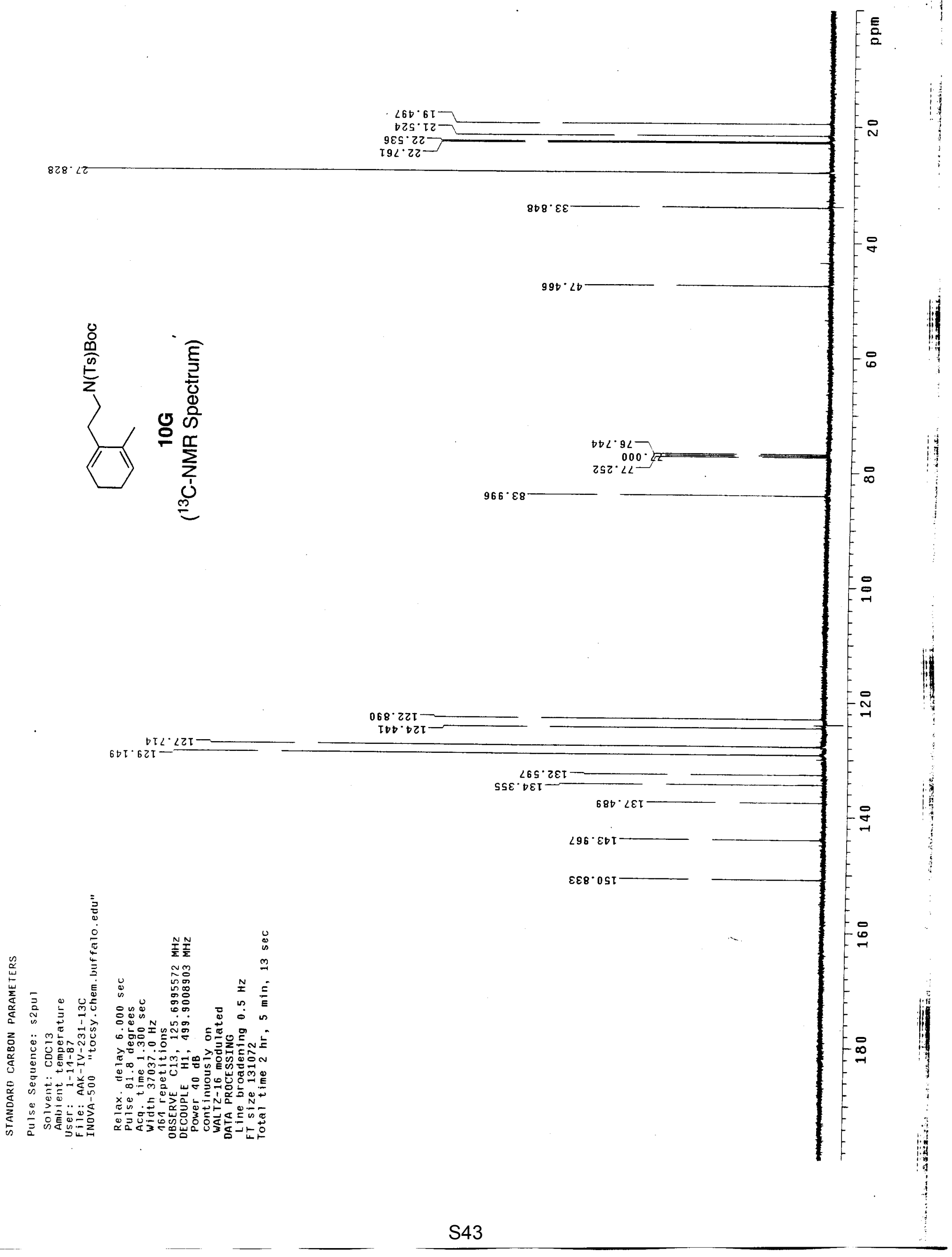




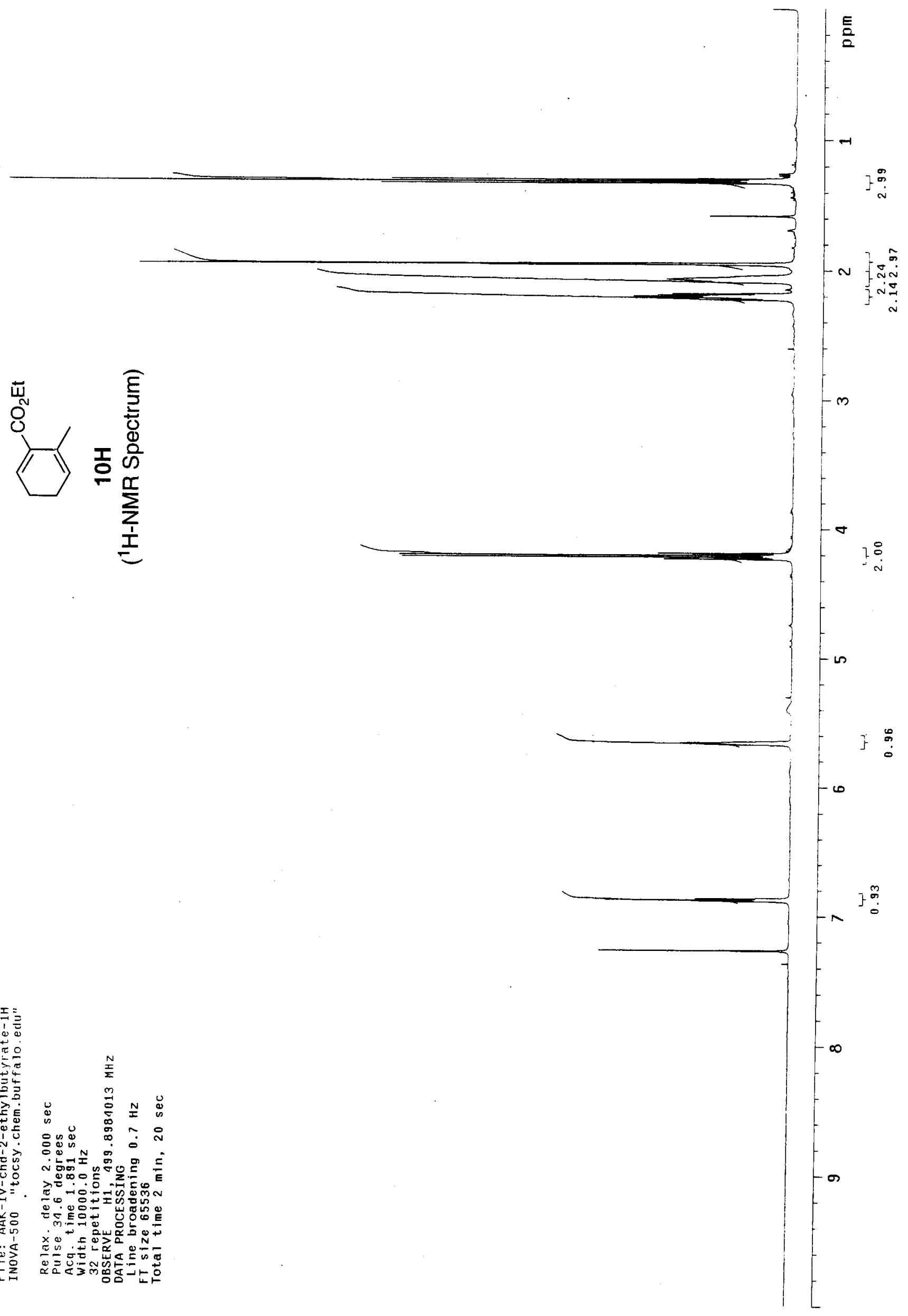

I

I

N

$\geq 2 \times 0=0$

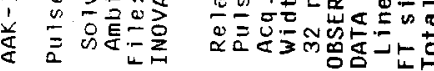




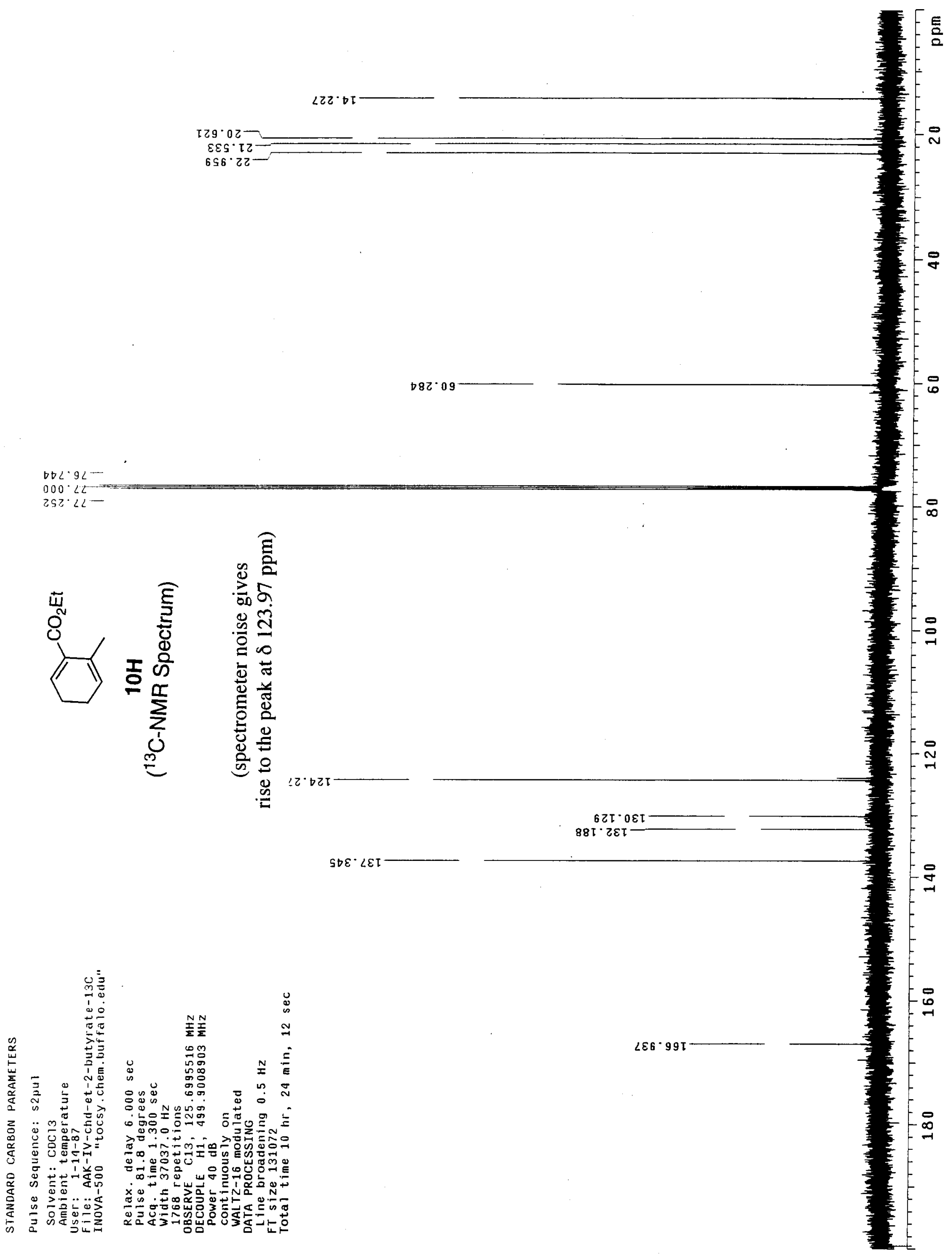




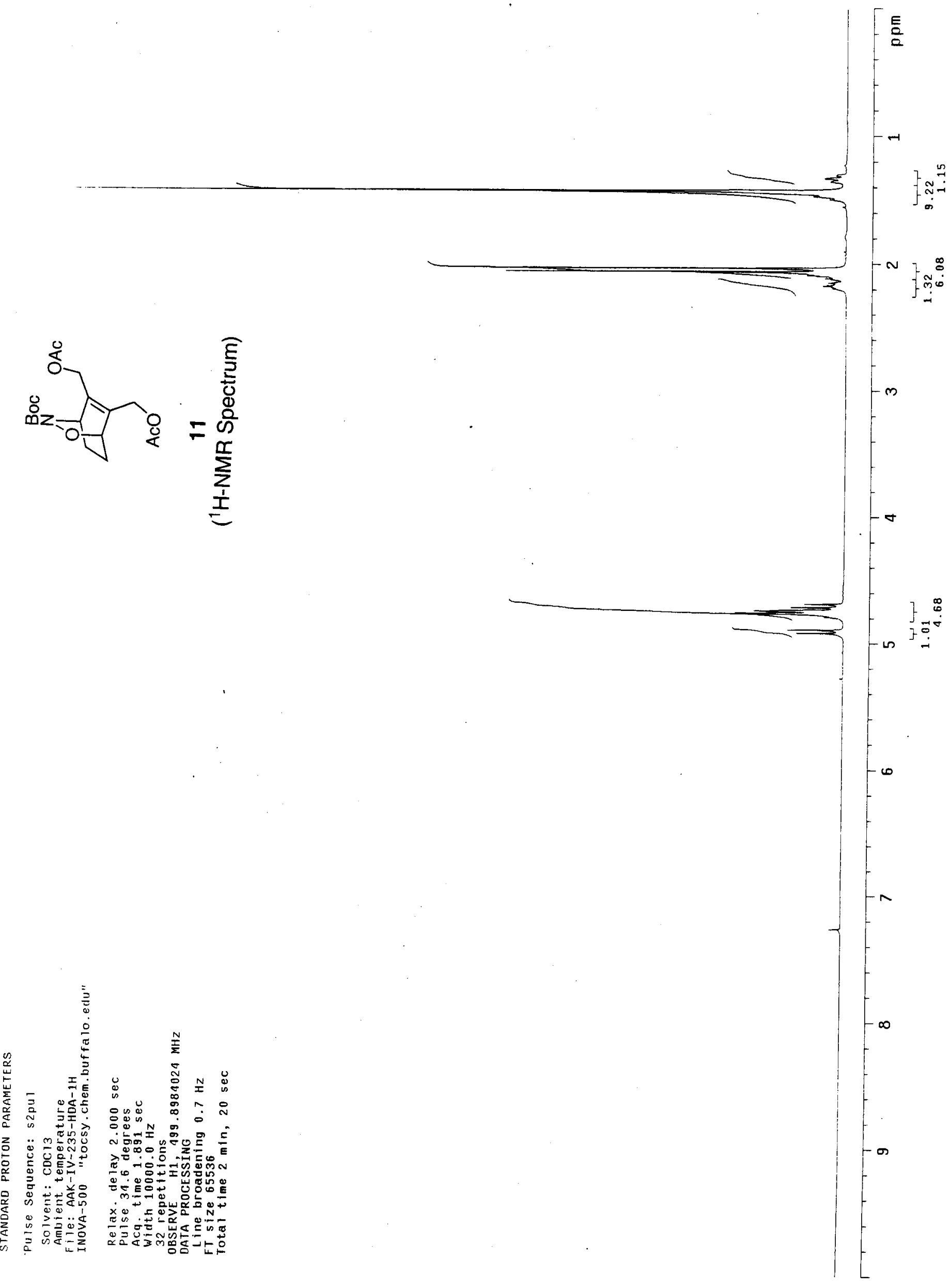




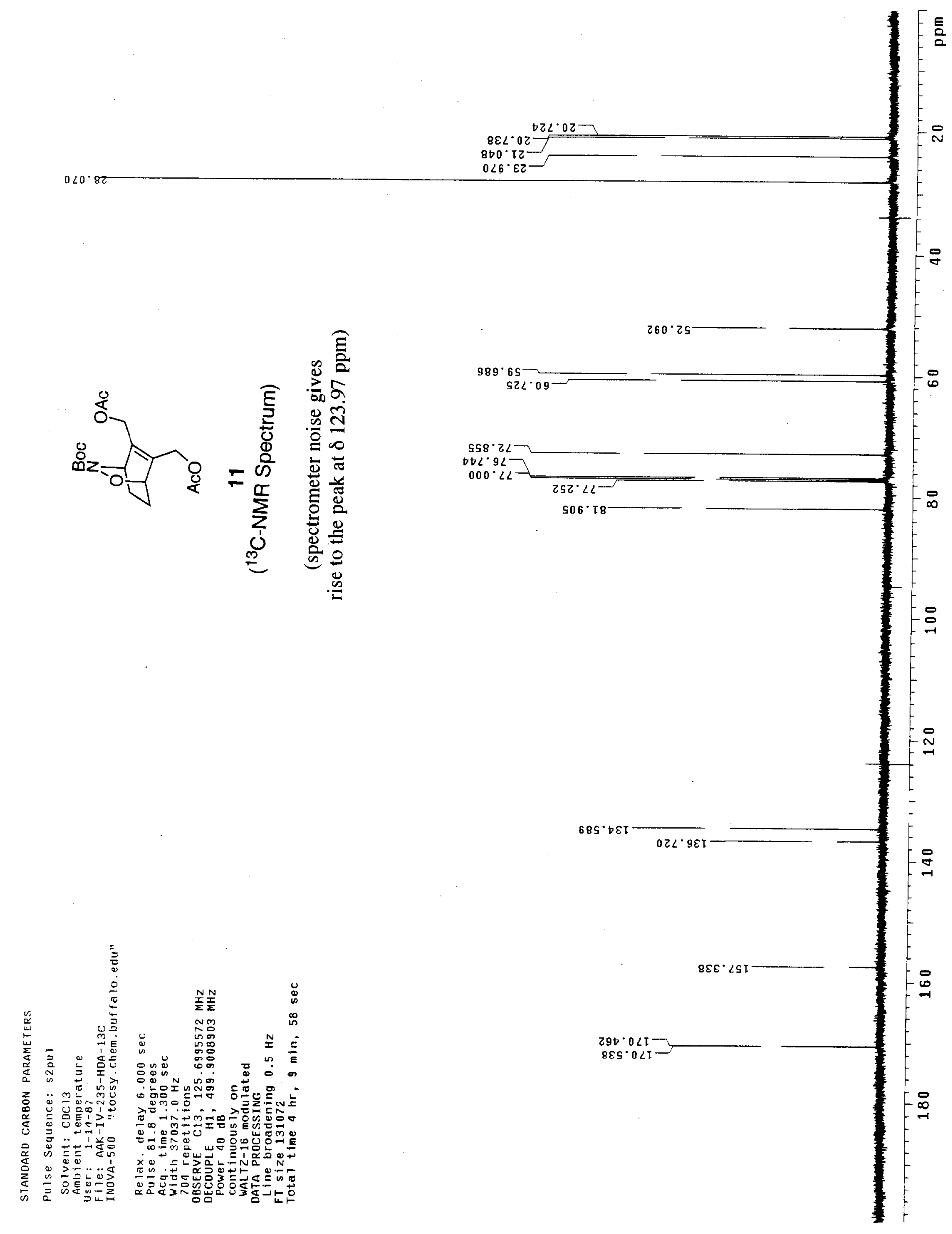




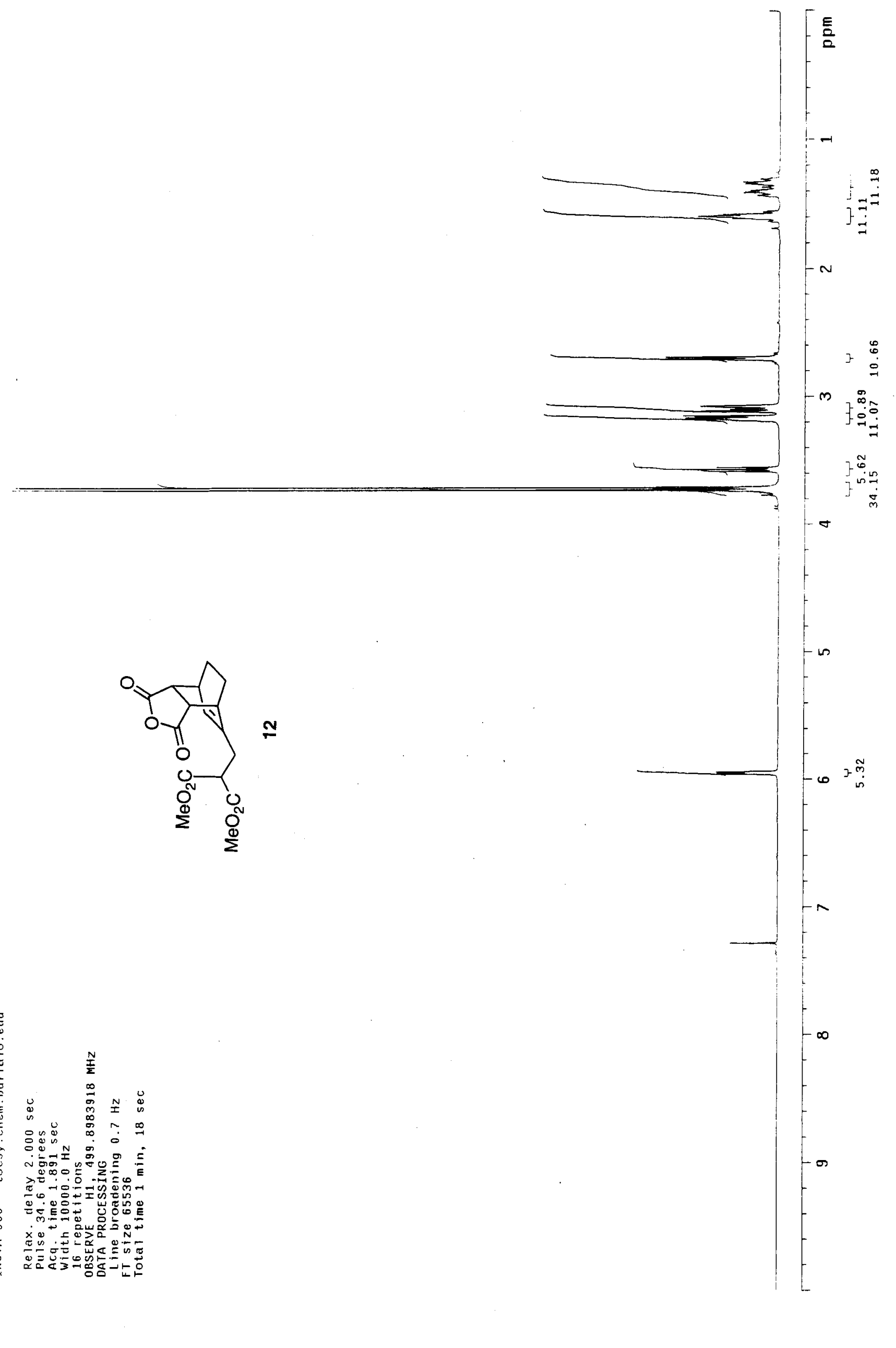




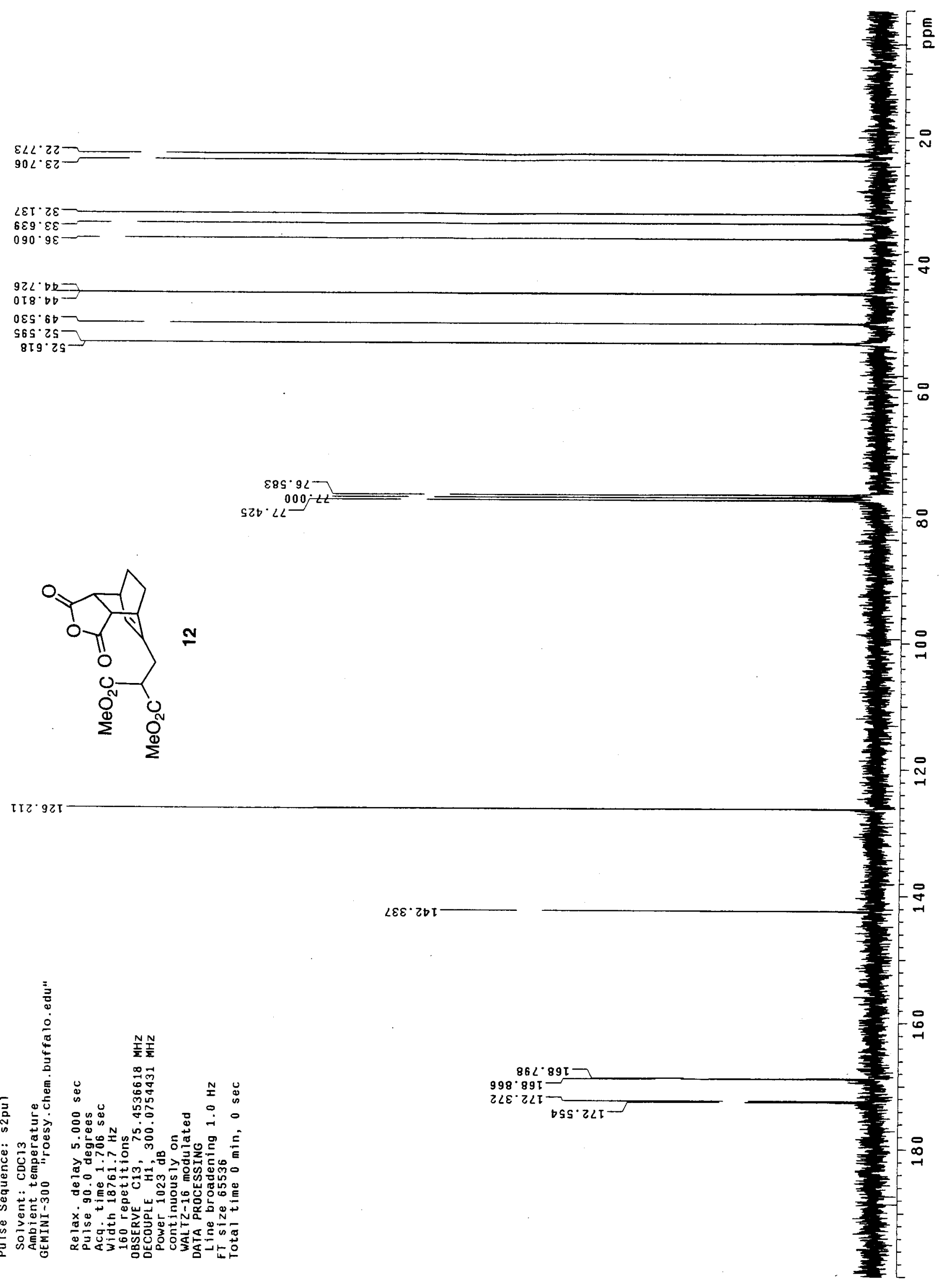

